\title{
Prices, Delay, and the Dynamics of Trade ${ }^{1}$
}

\author{
Diego Moreno \\ Departmento de Economia, Universidad Carlos III de Madrid, 28903 Getafe, Spain \\ dmoreno@eco.uc3m.es \\ and \\ John Wooders \\ Department of Economics, University of Arizona, Tucson, Arizona 85721; and \\ Department of Economics, Hong Kong University of Science and Technology, Hong Kong \\ jwooders@bpa.arizona.edu
}

\begin{abstract}
We characterize the dynamics of trading patterns and market composition when trade is bilateral, finding a trading partner is costly, prices are determined by bargaining, and preferences are private information. We show that equilibrium is inefficient and exhibits delay as sellers price discriminate between buyers with different values. As frictions vanish, transaction prices are asymptotically competitive and the welfare loss of inefficient trading approaches zero, even though the trading patterns continue to be inefficient and delay persists. Journal of Economic Literature Classification Numbers: D40, D50.

Key Words: decentralized markets; asymmetric information; trade dynamics; matching; bargaining; delay.
\end{abstract}

\section{INTRODUCTION}

In many markets, when (or whether) an agent trades, and at what price, depends on his own characteristics (his value, or the cost or quality of his

${ }^{1}$ We gratefully acknowledge financial support from the National Science Foundation, Grant SBR-9810481, and the Spanish Ministry of Education (DGES), Grant PB97-0091. We thank an associate editor and two referees for helpful suggestions. We benefited from comments by Eric Fisher, Roberto Serrano, Mark Walker, and the participants at seminars at Arizona State University, Boston University, California Institute of Technology, Cornell University, Institut d'Estudis Catalans (Barcelona Jocs), Universidad de Alicante, Universitat Pompeu Fabra, University of Bonn, ISER at Osaka University, the University of Tsukuba, and the participants at the 1999 Decentralization Conference, the 1999 Southern California Theory Conference, and the 1999 Stanford Institute for Theoretical Economics (SITE) conference. 
good), as well as on the characteristics of the other traders. In the market for new assistant professors of economics, for example, highly qualified job candidates tend to leave the market (i.e., to accept job offers) earlier than less well-qualified candidates. In the clothing market, high-value buyers purchase the new fall fashions as soon as the clothes enter stores, whereas low-value buyers purchase later in the season once the clothes go on sale. The distribution of the characteristics of active traders also varies over time: In the market for new assistant professors, for example, the proportion of active candidates that are highly qualified is larger when the market opens than when it closes. In these markets the "trading pattern" at each date (i.e., which types of buyers and sellers trade), and the "market composition" at each date (i.e., the characteristics of active traders) are determined endogenously and vary over time.

In this paper we introduce a simple model of a nonstationary dynamic market with heterogeneous traders in which trading patterns, market compositions, and transaction prices are determined endogenously. In the market there are two types of buyers, whose values are either "high" or "low," initially present in given proportions; all sellers can supply a unit of the good at equal cost. After the market opens there is no entry. Each period, active traders are randomly matched and bargain bilaterally. In the bargaining game one of the traders is randomly selected to make a take-it-or-leave-it price proposal. Bargaining is under incomplete information, as a seller does not know whether his partner has a high or a low value.

We first identify the trading patterns that arise in equilibrium and the dynamics of market composition: When cost is high, i.e., above the value of low-value buyers, but below the value of high-value buyers, the trading pattern is always separating (high-value buyers trade, but low-value buyers do not trade). When cost is low, i.e., below the values of both types of buyers, the case of primary interest, at most three (pure) trading patterns arise over the life of the market: in periods where high-value buyers are abundant (i.e., when their proportion exceeds a critical threshold we identify), the trading pattern is either separating or partially separating (high-value buyers trade, and low-value buyers trade only when they propose); in periods where high-value buyers are scarce, the trading pattern is pooling (both types of buyers trade). Moreover, the transitions from one trading pattern to the next are in a particular order: from separating to partially separating to pooling. When the market transits from one pure trading pattern to the next there may be a single intervening period in which the trading pattern is "mixed." In this case market equilibria are asymmetric. We establish that the proportion of high-value buyers in the market is decreasing over time, and that the trading pattern is eventually pooling. 
Our analysis reveals properties of market equilibria that are in sharp contrast with Walrasian equilibrium. Specifically, when cost is low and high-value buyers are abundant, the trading pattern is either separating or partially separating when the market opens. In this case, trading patterns are (constrained) inefficient. (Under efficient trading every match ends with trade-see below.) In addition, trade occurs only with delay: low-value buyers and sellers remain in the market longer, in expected terms, than they would if the trading patterns were efficient.

Inefficiency and delay arise as sellers price discriminate among heterogeneous buyers, regardless of whether information is complete or incomplete. Interestingly, as an example in Section 4 demonstrates, equilibrium surplus may be lower when information is complete rather than incomplete.

As frictions vanish, transaction prices converge to a competitive price, even though the trading patterns continue to be inefficient and delay persists. Nevertheless, since the length of delay is bounded, asymptotically the welfare loss of inefficient trading is zero and each trader obtains his competitive equilibrium utility.

\section{Related Literature}

Our results on trading patterns and their dynamics in nonstationary markets with heterogenous traders are novel and have no counterpart in the matching and bargaining literature. With the exceptions of Binmore and Herrero [1] and Peters [7], who study markets with a single type of buyer and a single type of seller, the matching and bargaining literature has focused on stationary equilibria (see, for example, Rubinstein and Wolinsky [8]).

Our findings that transaction prices are competitive as frictions vanish relate to results already in the literature. Gale [2], for example, obtains this result in a similar setting, except that information is complete. His work, however, does not address equilibrium dynamics or the efficiency of trading patterns, which are central issues in our work. In fact, markets exhibit interesting dynamics even when information is complete (see Section 4).

Serrano and Yosha [12] study whether transaction prices are competitive in a model inspired by Wolinsky [14]--see also Samuelson [9]. In their framework a trader may bargain either "tough" or "soft." If both traders in a match bargain tough, the outcome is no trade. Otherwise, the pair of bargaining positions determines at which of one of three exogenously given prices they trade. For the one-sided asymmetric information case (they also study the "two-sided" asymmetric information case, which we do not deal with here), they show that when cost is low and 
frictions are small, market equilibria are efficient and exhibit no delay. Price restrictions appear to play an important role in these results, which are at odds with those we obtain. In our model prices are fully endogenous; i.e., buyers and sellers can make arbitrary price offers.

There are several papers studying the efficiency of decentralized markets; see, e.g., Sattinger [10], Serrano and Yosha [11], and Jackson and Palfrey [4]. In a framework similar to ours, Jackson and Palfrey [4] show that there is a robust distribution of buyer and seller values for which equilibrium is inefficient for every bargaining game in a general class. Using Jackson and Palfrey's notion of efficiency, we show that although trading patterns may be inefficient, as frictions vanish the welfare loss due to inefficient trading vanishes. Thus, the simple bargaining game in which traders make take-it-or-leave-it price offers yields asymptotically efficient outcomes (despite the fact that trading patterns are inefficient). Hence Jackson and Palfrey's result loses significance when frictions are small.

Our work also relates to a large literature studying price dispersion and sales. Varian [13], for example, shows that sales provide a means for sellers to price discriminate between informed and uninformed consumers. In our model price discrimination arises as a consequence of the differential willingness of high- and low-value buyers to endure delay. This yields a remarkable parallel between seller behavior in our model and the behavior of a durable good monopolist--see, e.g., Hart and Tirole [3].

\section{THE MODEL}

A market for a single indivisible commodity operates from period 0 to $T$, where $T$ may be finite or infinite. At period $t=0$ there is a continuum of buyers and sellers, present in equal measures; no new traders enter the market subsequently. Each seller is endowed with a single unit of the indivisible good. Each buyer is endowed with one unit of money. Buyers and sellers preferences are characterized by, respectively, their values and costs: All sellers $(S)$ have the same cost, $c \geqslant 0$, whereas there are two types of buyers, "high-value" $(H)$ and "low-value" $(L)$, whose values are, respectively, $u^{H}$ and $u^{L}$, where $1 \geqslant u^{H}>u^{L} \geqslant 0$. We assume throughout that $u^{H}>c$. At date zero, high-value and low-value buyers are present in the population of buyers in proportions $b_{0}^{H} \in(0,1)$ and $b_{0}^{L}=1-b_{0}^{H}$, respectively. If a buyer whose value is $u^{\tau}$ trades with a seller at the price $p$ in time $t$, they obtain a utility of $\delta^{t}\left(u^{\tau}-p\right)$ and $\delta^{t}(p-c)$, respectively. Here $\delta \in(0,1)$, the discount factor, expresses the traders' impatience. ${ }^{2}$ A buyer or a seller who never trades obtains a utility of 0 .

\footnotetext{
${ }^{2}$ When $T$ is finite our results also hold for $\delta=1$.
} 
Each period every buyer (seller) remaining in the market meets a randomly selected seller (buyer) with probability $\alpha$, where $\alpha \in(0,1){ }^{3} \mathrm{~A}$ matched seller does not observe the buyer's value. When a buyer and a seller meet, one of them is selected randomly (with probability $\frac{1}{2}$ ) to propose a price at which to trade. If the proposed price is accepted by the other party, then the agents trade at that price and both leave the market. Otherwise, the agents remain in the market at the next date and wait for a new match. An agent who is not matched in the current period also remains in the market at the next date. A trader observes only the outcome of his own matches.

A strategy for a trader of type $\tau \in\{H, L, S\}$ is a pair $\left(p^{\tau}, r^{\tau}\right)$, where $p^{\tau}$ is a sequence (of real numbers) indicating the price offers the trader would make at each date if matched and selected to propose a price, and $r^{\tau}$ is a sequence of reservation prices specifying the maximum (minimum) price that a buyer (seller) would accept at each date if responding to a price offer. A strategy distribution is a triple $(p, r, \lambda)=\left[\left(p^{H_{i}}, r^{H_{i}}, \lambda^{H_{i}}\right)_{i=1}^{n^{H}},\left(p^{L_{i}}, r^{L_{i}}, \lambda^{L_{i}}\right)_{i=1}^{n^{L}}\right.$, $\left.\left(p^{S_{i}}, r^{S_{i}}, \lambda^{S_{i}}\right)_{i=1}^{n^{S}}\right]$, where for each $\tau \in\{H, L, S\}, \lambda^{\tau_{k}}>0$ is the proportion of type $\tau$ players using strategy $\left(p^{\tau_{k}}, r^{\tau_{k}}\right)$, and $n^{\tau}$ is the (countable) number of distinct strategies used by (a positive measure of) type $\tau$ traders. Note that $\sum_{k=1}^{n^{\tau}} \lambda^{\tau_{k}}=1$.

We do not restrict attention to symmetric strategy distributions (i.e., different agents of the same type may follow different strategies). Indeed, allowing asymmetric strategy distributions is necessary to guarantee existence of a market equilibrium-see the discussion on symmetry and the example provided in Moreno and Wooders [5, Section 4]. We consider only strategies in which a trader does not condition his actions in the current match on the history of his prior matches, but this restriction is inconsequential. ${ }^{4}$ For simplicity, we restrict attention to strategy distributions where only countably many distinct strategies are used. As we shall see, however, for discount factors near or equal to one, in equilibrium at most two different strategies are played by each type of trader.

\subsection{Laws of Motion}

Given a strategy distribution $(p, r, \lambda)$, for $\tau \in\{H, L, S\}$ and $k \leqslant n^{\tau}$ let $\lambda_{t}^{\tau_{k}}$ denote the proportion of agents following the $k$ th type $\tau$ strategy out of the

\footnotetext{
${ }^{3}$ We rule out the case $\alpha=1$ in order to ensure that a positive measure of each type of agent is present at all times, so that the laws of motion described in Section 2.1 are well defined.

${ }^{4}$ Since a trader only observes the outcomes of his own matches, his decision problem, and therefore his reservation price, is the same regardless of his history in prior matches-see Osborne and Rubinstein [6, pp. 154-162]. Thus, for any equilibrium in which the players' strategies depend on histories, there is another equilibrium in history-independent strategies resulting in identical transaction prices, trading patterns, and market compositions.
} 
total measure of agents of type $\tau$ who remain in the market at time $t$. (Throughout, we use $i, j$, and $k$, respectively, to index the strategies of buyers, sellers, and generic traders.) This proportion can be computed for $t \in\{0, \ldots, T\}$, given $\lambda_{0}^{\tau_{k}}=\lambda^{\tau_{k}}$, as

$$
\lambda_{t+1}^{\tau_{k}}=\frac{\lambda_{t}^{\tau_{k}}\left(1-\alpha z_{t}^{\tau_{k}}\right)}{\sum_{l=1}^{n^{\tau}} \lambda_{t}^{\tau_{l}}\left(1-\alpha z_{t}^{\tau_{l}}\right)}
$$

where $z_{t}^{\tau_{k}}$ denotes the probability that a trader who is matched at $t$ and who follows the strategy $\left(p_{t}^{\tau_{k}}, r_{t}^{\tau_{k}}\right)$ trades at $t$. (We shall also sometimes interpret $\lambda_{t}^{\tau_{k}}$ as a probability; e.g., $\lambda_{t}^{S_{f}}$ is the probability that a buyer matched at $t$ is matched with a seller following the $j$ th seller strategy.) The probability $z_{t}^{\tau_{k}}$ is computed as follows: For $x, y \in \mathbb{R}$ denote by $I(x, y)$ the indicator function, whose value is 1 if $x \geqslant y$, and 0 otherwise. Writing $B=\{H, L\}$ for the set of buyer types, then for $\tau \in B$ we have

$$
z_{t}^{\tau_{i}}=\frac{1}{2} \sum_{j=1}^{n^{s}} \lambda_{t}^{S_{j}} I\left(p_{t}^{\tau_{i}}, r_{t}^{S_{j}}\right)+\frac{1}{2} \sum_{j=1}^{n^{s}} \lambda_{t}^{S_{j}} I\left(r_{t}^{\tau_{i}}, p_{t}^{S_{j}}\right)
$$

For sellers, this probability is given by

$$
z_{t}^{S_{j}}=\frac{1}{2} \sum_{\tau \in B} b_{t}^{\tau} \sum_{i=1}^{n^{\tau}} \lambda_{t}^{\tau_{i}} I\left(r_{t}^{\tau_{i}}, p_{t}^{S_{j}}\right)+\frac{1}{2} \sum_{t \in B} b_{t}^{\tau} \sum_{i=1}^{n^{\tau}} \lambda_{t}^{\tau_{i}} I\left(p_{t}^{\tau_{i}}, r_{t}^{S_{j}}\right),
$$

where $b_{t}^{\tau}$, the proportion of the buyers of type $\tau$ out of the total measure of buyers remaining in the market at time $t$, can be computed for $t>0$, given $b_{0}^{\tau}$, as

$$
b_{t}^{\tau}=\frac{\left(1-\alpha z_{t-1}^{\tau}\right) b_{t-1}^{\tau}}{\left(1-\alpha z_{t-1}^{H}\right) b_{t-1}^{H}+\left(1-\alpha z_{t-1}^{L}\right) b_{t-1}^{L}},
$$

and $z_{t}^{\tau}=\sum_{i=1}^{n^{\tau}} \lambda_{t}^{\tau_{i}} z_{t}^{\tau_{i}}$ is the probability that a randomly selected type $\tau \in\{H, L, S\}$ trader who is matched at $t$ trades at $t$. Since there is a continuum of traders, the market evolves deterministically, even though a trader's own market experience is stochastic.

\subsection{Value Functions}

Given a strategy distribution $(p, r, \lambda)$, the expected utility at time $t$ of an agent of type $\tau \in\{H, L, S\}$ who is using strategy $\tau_{k}$ is

$$
V_{t}^{\tau_{k}}=\frac{\alpha}{2}\left(P_{t}^{\tau_{k}}+R_{t}^{t_{k}}\right)+(1-\alpha) \delta V_{t+1}^{\tau_{k}}
$$


In this expression, $P_{t}^{\tau_{k}}\left(R_{t}^{\tau_{k}}\right)$ is the expected utility to a trader of type $\tau$ following the $k$ th type $\tau$ strategy who is matched at $t$ and selected to propose (respond to) a price offer. These expected utilities can be calculated for $\tau \in B$ as

$$
P_{t}^{\tau_{i}}=\left(u^{\tau}-p_{t}^{\tau_{i}}\right) \sum_{j=1}^{n^{s}} \lambda_{t}^{S_{j}} I\left(p_{t}^{\tau_{i}}, r_{t}^{S_{j}}\right)+\left(1-\sum_{j=1}^{n^{s}} \lambda_{t}^{S_{j}} I\left(p_{t}^{\tau_{i}}, r_{t}^{S_{j}}\right)\right) \delta V_{t+1}^{\tau_{i}}
$$

and

$$
R_{t}^{\tau_{i}}=\sum_{j=1}^{n^{S}} \lambda_{t}^{S_{j}}\left(u^{\tau}-p_{t}^{S_{j}}\right) I\left(r_{t}^{\tau_{i}}, p_{t}^{S_{j}}\right)+\left(1-\sum_{j=1}^{n^{S}} \lambda_{t}^{S_{j}} I\left(r_{t}^{\tau_{i}}, p_{t}^{S_{j}}\right)\right) \delta V_{t+1}^{\tau_{i}}
$$

For sellers we have

$$
\begin{aligned}
P_{t}^{S_{j}}= & \left(p_{t}^{S_{j}}-c\right) \sum_{\tau \in B} b_{t}^{\tau} \sum_{i=1}^{n^{\tau}} \lambda_{t}^{\tau_{i}} I\left(r_{t}^{\tau_{i}}, p_{t}^{S_{j}}\right) \\
& +\left(1-\sum_{\tau \in B} b_{t}^{\tau} \sum_{i=1}^{n^{\tau}} \lambda_{t}^{\tau_{i}} I\left(r_{t}^{\tau_{i}}, p_{t}^{S_{j}}\right)\right) \delta V_{t+1}^{S_{j}}
\end{aligned}
$$

and

$$
\begin{aligned}
R_{t}^{S_{j}}= & \sum_{\tau \in B} b_{t}^{\tau} \sum_{i=1}^{n^{\tau}} \lambda_{t}^{\tau_{i}}\left(p_{t}^{\tau_{i}}-c\right) I\left(p_{t}^{\tau_{i}}, r_{t}^{S_{j}}\right) \\
& +\left(1-\sum_{\tau \in B} b_{t}^{\tau} \sum_{i=1}^{n^{\tau}} \lambda_{t}^{\tau_{i}} I\left(p_{t}^{\tau_{i}}, r_{t}^{s_{j}}\right)\right) \delta V_{t+1}^{S_{j}}
\end{aligned}
$$

When $T$ is finite, these expected utilities are calculated using $V_{T+1}^{\tau_{k}}=0$ for $\tau \in\{H, L, S\}$ and $k \leqslant n^{\tau}$.

\subsection{Equilibrium}

A strategy distribution $(p, r, \lambda)$ is a market equilibrium if for each $t \epsilon$ $\{0, \ldots, T\}$, each $\tau \in B$ and $i \in\left\{1, \ldots, n^{\tau}\right\}$, and each $j \in\left\{1, \ldots, n^{s}\right\}$

$$
\begin{gathered}
u^{\tau}-r_{t}^{\tau_{i}}=\delta V_{t+1}^{\tau_{i}}, \\
r_{t}^{S_{j}}-c=\delta V_{t+1}^{S_{j}},
\end{gathered}
$$


and

$$
\begin{aligned}
& p_{t}^{\tau_{i}} \in \underset{x}{\arg \max }\left(u^{\tau}-x\right) \sum_{j=1}^{n^{S}} \lambda_{t}^{S_{j}} I\left(x, r_{t}^{S_{j}}\right)+\left(1-\sum_{j=1}^{n^{S}} \lambda_{t}^{S_{j}} I\left(x, r_{t}^{S_{j}}\right)\right) \\
& p_{t}^{S_{j}} \in \underset{x}{\arg \max }(x-c) \sum_{\tau \in B} b_{t}^{\tau} \sum_{i=1}^{n^{\tau}} \lambda_{t}^{\tau_{i}} I\left(r_{t}^{\tau_{i}}, x\right) \\
& +\left(1-\sum_{\tau \in B} b_{t}^{\tau} \sum_{i=1}^{n^{\tau}} \lambda_{t}^{\tau_{i}} I\left(r_{t}^{\tau_{i}}, x\right)\right) \delta V_{t+1}^{S_{j}} .
\end{aligned}
$$

Condition (E.1) requires that at each date a trader's reservation price makes him indifferent between accepting or rejecting an offer of his reservation price. Condition (E.1) builds perfection into our equilibrium notion by requiring that an agent accepts any price offer which gives him a higher utility that he would obtain by rejecting the offer. Condition (E.2) ensures that price offers are optimal. Given the recursive nature of our setting, in a market equilibrium traders' strategies are globally optimal; i.e., no trader can do better by changing his reservation prices or price offers simultaneously at more than one date.

In a market equilibrium, traders form their expectations of the proportion of buyers of each type remaining in the market $\left(b_{t}^{\tau}\right)$, and the proportion of traders following each of the strategies being played $\left(\lambda_{t}^{\tau_{k}}\right)$, on the basis of the strategy distribution being played. Moreover, each trader maximizes his expected utility at each of his information sets. Thus, the notion of market equilibrium is in the spirit of sequential (or Bayes perfect) equilibrium.

Existence of market equilibria under general conditions is established in Moreno and Wooders [5] by means of a fixed point argument. It might seem that if $T$ were finite one could calculate a market equilibrium via backward induction. Computing a traders' reservation price and optimal price offer at a date $t$, however, requires knowing the market composition (i.e., the proportion of traders of each type present in the market) at $t$, as well as his expected utility if he remains in the market at $t+1$. Since the market composition at date $t$ is determined by the trading patterns (and the traders' strategies) prior to $t$, a market equilibrium cannot be computed by backward induction.

\subsection{Trading Patterns and Efficiency}

Characterizing market equilibria requires determining the "trading patterns" that may arise. We classify the matches into four types, depending upon the buyer's value and who proposes: (i) a high-value buyer proposes (to a seller), (ii) a low-value buyer proposes, (iii) a seller proposes to a 
high-value buyer, and (iv) a seller proposes to a low-value buyer. A trading pattern at a given date $t$ specifies the proportion of each type of match that ends with trade, and is represented by a vector $z_{t}=\left(z_{t}^{H S}, z_{t}^{L S}, z_{t}^{S H}, z_{t}^{S L}\right) \in$ $[0,1]^{4}$. Given a strategy distribution, these proportions are

$$
z_{t}^{\tau S}=\sum_{i=1}^{n^{\tau}} \lambda_{t}^{\tau_{i}} \sum_{j=1}^{n^{S}} \lambda_{t}^{S_{j}} I\left(p_{t}^{\tau_{i}}, r_{t}^{S_{j}}\right),
$$

and

$$
z_{t}^{S \tau}=\sum_{j=1}^{n^{S}} \lambda_{t}^{S_{j}} \sum_{i=1}^{n^{\tau}} \lambda_{t}^{\tau_{i}} I\left(r_{t}^{\tau_{i}}, p_{t}^{S_{j}}\right) .
$$

Note that $z_{t}^{\tau}=\left(z_{t}^{\tau S}+z_{t}^{S \tau}\right) / 2$ (see Section 2.1 above). Also note that the set of possible trading patterns does not depend upon whether values are private information. In a "pure" trading pattern every match of the same type has the same outcome (either trade or no trade); i.e., the pure trading patterns are the elements of $[0,1]^{4}$ whose coordinates are either zeros or ones. (Hence there are 16 possible pure trading patterns.) All other trading patterns are "mixed."

The surplus realized is determined by the sequence of trading patterns. Given a sequence $z=\left\{z_{t}\right\}_{t=0}^{T}$ specifying the trading pattern at each date, the surplus $G(\delta, T, z)$ is given by

$$
G(\delta, T, z)=b_{0}^{H}\left(u^{H}-c\right) g^{H}(\delta, T, z)+b_{0}^{L}\left(u^{L}-c\right) g^{L}(\delta, T, z),
$$

where for $\tau \in\{H, L\}$

$$
\begin{aligned}
g^{\tau}(\delta, T, z)= & \alpha z_{0}^{\tau}+\delta\left(1-\alpha z_{0}^{\tau}\right) \alpha z_{1}^{\tau}+\cdots \\
& +\delta^{T}\left(1-\alpha z_{0}^{\tau}\right)\left(1-\alpha z_{1}^{\tau}\right) \cdots\left(1-\alpha z_{T-1}^{\tau}\right) \alpha z_{T}^{\tau} .
\end{aligned}
$$

In this expression, $b_{0}^{\tau}\left(u^{\tau}-c\right) g^{\tau}(\delta, T, z)$ is the surplus realized in matches of type $\tau$ buyers and sellers.

In order to evaluate the welfare properties of alternative market outcomes, it is useful to have a notion of efficiency. Following Jackson and Palfrey [4], we say that a sequence of trading patterns is (constrained) efficient if it maximizes surplus on the set of all sequences of trading patterns. In the high cost case $\left(c>u^{L}\right)$ the unique efficient sequence of trading patterns is separating for each $t$; i.e., for each $t, z_{t}^{H S}=z_{t}^{S H}=1$ and $z_{t}^{L S}=$ $z_{t}^{S L}=0$, whereas in the low cost case $\left(c<u^{L}\right)$ the unique efficient sequence of trading patterns is pooling; i.e., for each $t, z_{t}^{H S}=z_{t}^{S H}=z_{t}^{L S}=z_{t}^{S L}=1$. 


\section{PROPERTIES OF MARKET EQUILIBRIA}

In this section we identify the equilibrium trading patterns and their dynamics, and study the properties of market equilibria as frictions vanish. We begin with the high-cost case.

\subsection{High Cost}

Supply and demand schedules in this case are illustrated below in Fig. 1a $\left(c>u^{L}\right)$. Beginning with this case allows us to discuss the workings of our model in a simple environment and facilitates understanding the subtleties that arise in the more interesting case where there are gains to trade between sellers and both types of buyers.

(a)

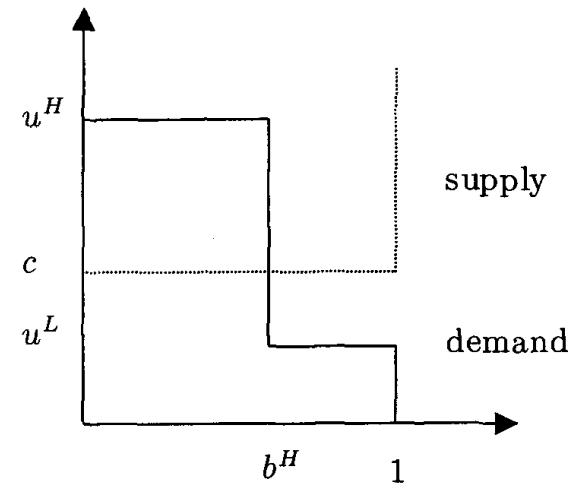

(b)

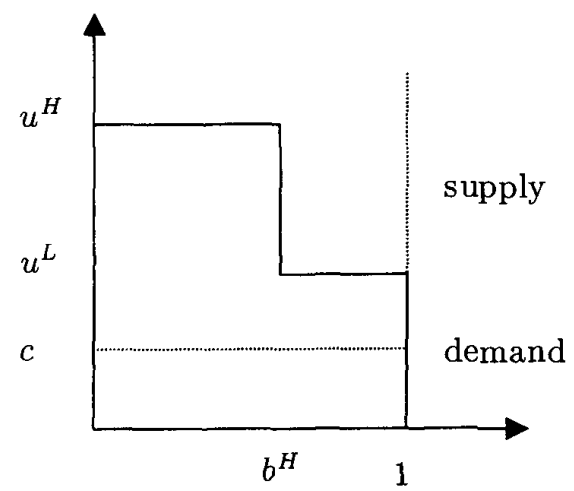

FIG. 1. (a) Supply and demand in the high-cost case. (b) Supply and demand in the low-cost case. 
Market equilibria in this case have a simple structure: at every date highvalue (low-value) buyers offer a price equal to (below) the seller reservation price, and sellers offer a price equal to the high-value-buyer reservation price. Thus, only high-value buyers, and the sellers they are matched with, trade. Since the trading pattern is separating at each date, the market equilibrium is constrained efficient. When traders are sufficiently patient (i.e., $\delta$ is close to 1) and the time horizon is sufficiently long, transaction prices are close to the competitive price (i.e., $c$ ), and surplus approaches the competitive surplus-equal to $b_{0}^{H}\left(u^{H}-c\right)$. Intuitively this is because when the time horizon is long, high-value buyers eventually become scarce and the seller-reservation price approaches $c$. If high-value buyers are patient, their reservation price (at early dates) is also close to $c$. These findings are summarized in Proposition 1. We provide an informal discussion of these results when $T<\infty$.

Let $(p, r, \lambda)$ be a market equilibrium. As an agent who does not trade while the market is open obtains a utility of zero (i.e., $V_{T+1}^{\tau}=0$ ), by (E.1) reservation prices at the last date are $r_{T}^{H}=u^{H}, r_{T}^{L}=u^{L}$, and $r_{T}^{S}=c$. Hence $r_{T}^{H}>r_{T}^{S}>r_{T}^{L}$. It is easy to see that high-value (low-value) buyers offer at date $T$ a price equal to (below) the seller-reservation price: A high-value (low-value) buyer obtains a utility of $u^{H}-r_{T}^{S}=u^{H}-c>0\left(u^{L}-r_{T}^{S}=\right.$ $\left.u^{L}-c<0\right)$ offering $r_{T}^{S}$, the lowest price accepted by sellers, and obtains $\delta V_{T+1}^{H}=0\left(\delta V_{T+1}^{L}=0\right)$ with a lower price offer. Thus, $p_{T}^{H}=r_{T}^{S}\left(p_{T}^{L}<r_{T}^{S}\right)$. Sellers offer at date $T$ the high-value-buyer reservation price (i.e., the highest price accepted by high-value buyers): a seller who offers $r_{T}^{H}$ obtains an expected utility of $b_{T}^{H}\left(r_{T}^{H}-c\right)=b_{T}^{H}\left(u^{H}-c\right)>0$, whereas he obtains $r_{T}^{L}-c=u^{L}-c<0$ offering $r_{T}^{L}$. Note that $b_{T}^{H}>0$ since a measure $(1-\alpha)^{T} b_{0}^{H}>0$ of high-value buyers has never been matched before $T^{5}$ Thus, $p_{T}^{S}=r_{T}^{H}$. Hence the pattern of trade at date $T$ is separating.

Traders' expected utilities at $T$ are $V_{T}^{H}=\frac{1}{2} \alpha\left(u^{H}-c\right), V_{T}^{L}=0$, and $V_{T}^{S}=$ $\frac{1}{2} \alpha b_{T}^{H}\left(u^{H}-c\right)$. Using (E.1) again we calculate traders' reservation prices at $T-1$ to obtain $r_{T-1}^{H}=u^{H}-\delta \frac{1}{2} \alpha\left(u^{H}-c\right), r_{T-1}^{S}=c+\delta \frac{1}{2} \alpha b_{T}^{H}\left(u^{H}-c\right)$, and $r_{T-1}^{L}=u^{L}$. Thus $r_{T-1}^{H}>r_{T-1}^{S}>r_{T-1}^{L}$, regardless of the value of $b_{T}^{H}$, and the same pattern of trade arises at date $T-1$. In fact, it can be shown by induction that reservation prices satisfy this inequality at every date $t$, independently of $b_{t}^{H}$, and therefore that the pattern of trade is separating at every date. Given the initial proportion of high-value buyers in the market and knowing the pattern of trade at each date, we can compute the entire evolution of

\footnotetext{
${ }^{5}$ It is interesting to observe that for $\alpha=1$ (and $T \geqslant 1$ ), a case we have ruled out, there are multiple (even asymmetric) market equilibria. In an equilibrium all high-value buyers trade at date zero; thus, at subsequent dates any price offer above $c$ (in fact, above $u^{L}$ ) is optimal for sellers. This leads to the existence of multiple equilibria that differ in transaction prices. As $\delta$ approaches 1 and $T$ approaches infinity, there continue to be multiple equilibria, but transaction prices approach the competitive price.
} 
the market composition (i.e., the sequence $\left\{b_{t}^{H}\right\}_{t=0}^{T}$ ). Knowing the trading pattern and the market composition at each date, the vector of reservation prices is computed recursively. Transaction prices are the seller-reservation price when high-value buyers propose, and the high-value-buyer reservation price when sellers propose.

For each $\delta \in(0,1), T$, and $\tau \in\{H, S\}$, let $r^{\tau}(\delta, T)$ be a sequence of equilibrium reservation prices.

Proposition 1. Assume that $c>u^{L}$. Let $(p, r, \lambda)$ be a market equilibrium, and let $\bar{t} \in\{0, \ldots, T\}$.

Reservation prices:

(P1.1.1) $\quad r_{i}^{\tau_{i}}=r_{i}^{\tau}$ for every $\tau \in\{H, L, S\}$ and $i \leqslant n^{\tau}$.

(P1.1.2) $\quad r_{i}^{H}>r_{i}^{S}>r_{\bar{i}}^{L}$.

Price offers:

(P1.2) $p_{\bar{i}}^{H_{i}}=r_{i}^{S}$ for $i \leqslant n^{H}, p_{\bar{t}}^{L_{i}}<r_{i}^{S}$ for $i \leqslant n^{L}$, and $p_{\bar{i}}^{S_{j}}=r_{\dot{t}}^{H}$ for $j \leqslant n^{S}$.

Market composition:

(P1.3) $\quad b_{i+1}^{H}=\frac{(1-\alpha) b_{i}^{H}}{(1-\alpha) b_{i}^{H}+1-b_{i}^{H}}<b_{i}^{H}$.

Equilibrium surplus:

(P1.4) $G(\delta, T)=b_{0}^{H}\left(u^{H}-c\right) \frac{\alpha\left(1-\delta^{T+1}(1-\alpha)^{T+1}\right)}{1-\delta(1-\alpha)}$.

Transaction prices and surplus as frictions vanish:

(P1.5) If $T<\infty$, then

(P1.5.1) $\lim _{\delta \rightarrow 1} \lim _{T \rightarrow \infty} r_{i}^{\tau}(\delta, T)=\lim _{T \rightarrow \infty} \lim _{\delta \rightarrow 1} r_{i}^{\tau}(\delta, T)=c$, for $\tau \in\{H, S\}$.

(P1.5.2) $\lim _{\delta \rightarrow 1} \lim _{T \rightarrow \infty} G(\delta, T)=\lim _{T \rightarrow \infty} \lim _{\delta \rightarrow 1} G(\delta, T)=b_{0}^{H}\left(u^{H}-c\right)$.

(P1.6) If $T=\infty$, then

(P1.6.1) $\lim _{\delta \rightarrow 1} r_{i}^{\tau}(\delta, T)=c$, for $\tau \in\{H, S\}$.

(P1.6.2) $\lim _{\delta \rightarrow 1} G(\delta, T)=b_{0}^{H}\left(u^{H}-c\right)$.

Figure 2 below shows equilibrium transaction prices for a market in which cost is high. The mean transaction price (weighted by the volume of trade) is 0.4308 , which is near reservation prices in the first few periods since most trade occurs within the first few periods. In the competitive equilibrium of this market the price is 0.2 and the entire surplus of 0.7520 goes to high-value buyers. In contrast, in the market equilibrium sellers capture $29 \%$ of the total (discounted) surplus of 0.6834 , despite the fact that frictions are relatively small (the probability that an agent is never matched is $\alpha^{T+1}=\frac{1}{1024}$. Even as $\delta$ approaches one sellers capture $15 \%$ of the total surplus of 0.7513 . 


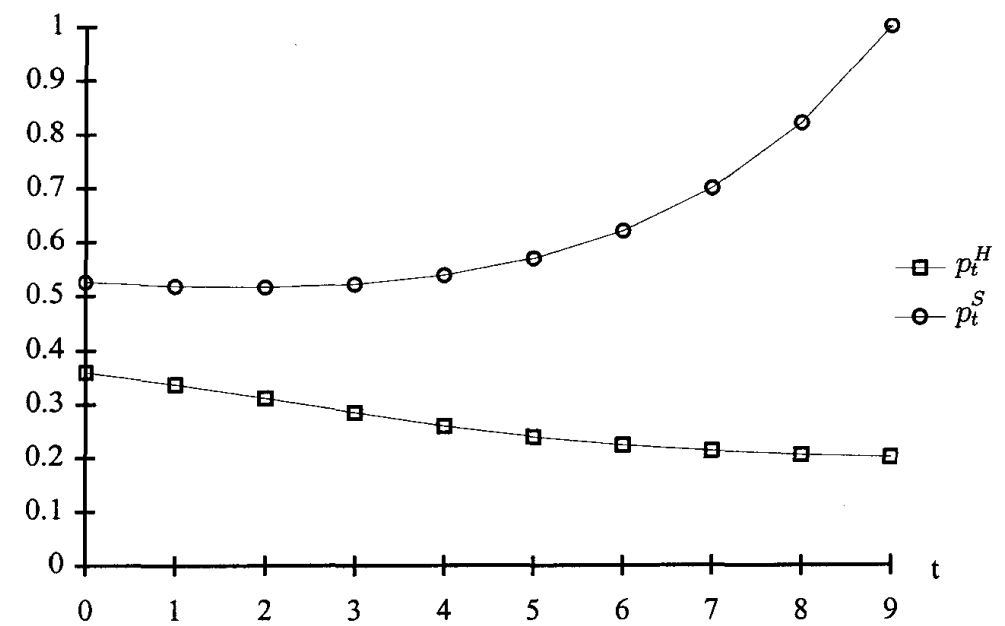

FIG. 2. Equilibrium in the high-cost case $\left(b_{0}^{H}=0.94, u^{H}=1, u^{L}=0.1, c=0.2, \delta=0.9\right.$, $\alpha=0.5$ ).

\subsection{Low Cost}

Figure $1 \mathrm{~b}$ illustrates the supply and demand schedules for the low-cost case $\left(c<u^{L}\right)$. To discuss the difficulties that arise in the analysis, assume that in a market equilibrium (i) traders of the same type have the same reservation price, (ii) sellers offer either the high-value-buyer reservation price $r_{t}^{H}$ or the low-value-buyer reservation price $r_{t}^{L}$ and (iii) $r_{t}^{H}>$ $\max \left\{r_{t}^{L}, r_{t}^{S}\right\}$. (See Proposition 2 below.) When a seller offers $r_{t}^{H}$ at date $t$, he trades only with high-value buyers and obtains an expected utility of

$$
b_{t}^{H}\left(r_{t}^{H}-c\right)+\left(1-b_{t}^{H}\right)\left(r_{t}^{S}-c\right) .
$$

(Recall that $\delta V_{t+1}^{S}=r_{t}^{S}-c$ by (E.1).) A seller who offers $r_{t}^{L}$ at date $t$ trades with both types of buyers, and obtains $r_{t}^{L}-c$. Therefore it is optimal for a seller to offer the high-value-buyer reservation price if

$$
b_{t}^{H}\left(r_{t}^{H}-r_{t}^{S}\right) \geqslant r_{t}^{L}-r_{t}^{S} .
$$

In other words, sellers offer the high-value-buyer reservation price if the probability that the current partner is a high-value buyer times the gains to trade with high-value buyers is greater than the gains to trade with lowvalue buyers. (In both cases, the gains are calculated relative to the reservation prices, rather than the actual values or costs.) Writing $\pi_{t}$ for the ratio $\left(r_{t}^{L}-r_{t}^{S}\right) /\left(r_{t}^{H}-r_{t}^{S}\right)$, which measures the relative gains to trade of sellers 
with low-value buyers versus high-value buyers, the inequality above can be written as

$$
b_{t}^{H} \geqslant \pi_{t}
$$

Hence, in contrast to the low cost case where the trading pattern is separating regardless of the market composition, in the present case the trading pattern at date $t$ depends on the market composition. Further, the market composition at date $t$ is determined in turn by the trading patterns prior to $t$. Thus, the entire sequence of trading patterns and market compositions must be determined simultaneously.

\section{Trading Patterns and Their Dynamics}

Proposition 2 establishes some basic facts about equilibrium price offers and reservation prices. Using these facts we identify the trading patterns that arise in equilibrium, as well as their dynamics.

Proposition 2. Assume that $c<u^{L}$. Let $(p, r, \lambda)$ be a market equilibrium and let $\bar{t} \in\{0, \ldots, T\}$.

\section{Reservation prices:}

(P2.1.1) $\quad r_{\bar{t}}^{\tau_{i}}=r_{i}^{\tau}$ for every $\tau \in\{H, L, S\}$ and $i \leqslant n^{\tau}$.

(P2.1.2) $\quad r_{i}^{H}>\max \left\{r_{\bar{t}}^{L}, r_{i}^{S}\right\}$.

High-value-buyer price offers:

(P2.2) $p_{\bar{i}}^{H_{i}}=r_{\bar{i}}^{S}$, for every $i \leqslant n^{H}$.

Low-value-buyer price offers:

(P2.3.1) $p_{i}^{L_{i}} \leqslant r_{\bar{i}}^{S}$ for every $i \leqslant n^{L}$.

(P2.3.2) Assume $T<\infty$; there is $\varepsilon(\alpha, T)>0$ such that if $\delta>1-\varepsilon(\alpha, T)$, then

(i) $p_{\bar{t}}^{L_{i}}<r_{\bar{t}}^{S}$ for some $i \leqslant n^{L}$ implies $p_{t}^{L_{i}}<r_{t}^{S}$ for every $t<\bar{t}$ and $i \leqslant n^{L}$.

(ii) $p_{t}^{L_{i}}=r_{i}^{S}$ for some $i \leqslant n^{L}$ implies $p_{t}^{L_{i}}=r_{t}^{S}$ for every $t>\bar{t}$ and $i \leqslant n^{L}$.

Seller price offers:

(P2.4.1) $p_{\bar{t}^{j}}^{S_{j}}\left\{\left\{r_{\bar{t}}^{L}, r_{i}^{H}\right\}\right.$ for every $j \leqslant n^{S}$.

(P2.4.2) If $p_{\bar{t}}^{S_{j}}=r_{\bar{t}}^{L}$ for some $j \leqslant n^{S}$, then $p_{t}^{S_{j}}=r_{t}^{L}$ for every $t>\bar{t}$ and $j \leqslant n^{S}$.

(P2.4.3) If $p_{\bar{t}}^{S_{j}}=r_{\bar{i}}^{H}$ for some $j \leqslant n^{S}$, then $p_{t}^{S_{j}}=r_{t}^{H}$ for every $t<\bar{t}$ and $j \leqslant n^{S}$. 
Seller and low-value-buyer price offers:

(P2.5) If $p_{i}^{S_{i}}=r_{\bar{t}}^{L}$ for some $j \leqslant n^{S}$, then $p_{\bar{i}}^{L_{i}}=r_{\bar{t}}^{S}$ for every $i \leqslant n^{L}$.

Proposition 2 implies that at most three pure trading patterns may arise in equilibrium: by (P2.2) every match where a high-value buyer proposes ends with trade (i.e., $z_{t}^{H S}=1$ for all $t$ ), and by $(\mathrm{P} 2.1 .2)$ and $(\mathrm{P} 2.4 .1)$ every match where a seller proposes to a high-value buyer also ends with trade (i.e., $z_{t}^{S H}=1$ for all $t$ ). In addition, (P2.5) rules out the trading patterns in which matches between sellers and low-value buyers end with trade when the seller proposes, but end without trade when the buyer proposes (i.e., $z_{t}^{S L}=1$ implies $z_{t}^{L S}=1$ ). Thus, only three pure trading patterns may arise in equilibrium: separating ( $\mathrm{S}$ ), that is, all matches between high-value buyers and sellers end with trade, whereas all matches between low-value buyers and sellers end without trade (i.e., $z_{t}^{H S}=z_{t}^{S H}=1$, and $z_{t}^{L S}=z_{t}^{S L}=0$ ); partially separating (PS), that is, all matches between high-value buyers and sellers end with trade and matches between low-value buyers and sellers end with trade only if the buyer proposes (i.e., $z_{t}^{H S}=z_{t}^{S H}=z_{t}^{L S}=1$, and $z_{t}^{S L}=0$ ); and pooling $(\mathrm{P})$, that is, all matches end with trade (i.e., $\left.z_{t}^{H S}=z_{t}^{S H}=z_{t}^{L S}=z_{t}^{S L}=1\right)$. The relation between price offers and reservation prices in each of these trading patterns are summarized in Table I.

Mixed trading patterns are not ruled out by Proposition 2. Since all matches between high-value buyers and sellers end with trade, if two matches of the same type have different outcomes, they must be matches of a low-value buyer and a seller. Moreover, by (P2.5) if a positive proportion of matches where a seller proposes to a low-value buyer end with trade, then all matches where a low-value buyer proposes end with trade (i.e., $z_{t}^{S L}>0$ implies $\left.z_{t}^{L S}=1\right)$. Hence only mixed trading patterns of the form $z_{t}^{H S}=z_{t}^{S H}=1, z_{t}^{L S} \in(0,1)$ and $z_{t}^{S L}=0$, referred to as "S-PS," and $z_{t}^{H S}=z_{t}^{S H}=z_{t}^{L S}=1, z_{t}^{S L} \in(0,1)$, referred to as "PS-P," may arise in equilibrium. Proposition 2 ensures that a PS-P trading pattern arises in at most one period (P2.4.3). Moreover, when $T$ is finite and the discount factor is sufficiently high, a S-PS trading pattern arises in at most one period,

TABLE I

Equilibrium Pure Trading Patterns when $c<u^{L}$

\begin{tabular}{lccc}
\hline \multirow{2}{*}{ Trading patterns } & \multicolumn{3}{c}{ Price offers } \\
\cline { 2 - 4 } & Sellers & High value & Low value \\
\hline Separating & $p_{t}^{S}=r_{t}^{H}$ & $p_{t}^{H}=r_{t}^{S}$ & $p_{t}^{L}<r_{t}^{S}$ \\
Partially separating & $p_{t}^{S}=r_{t}^{H}$ & $p_{t}^{H}=r_{t}^{S}$ & $p_{t}^{L}=r_{t}^{S}$ \\
Pooling & $p_{t}^{S}=r_{t}^{L}$ & $p_{t}^{H}=r_{t}^{S}$ & $p_{t}^{L}=r_{t}^{S}$ \\
\hline
\end{tabular}


since by (P2.3.2) once a positive proportion of low-value buyers offer the seller reservation price, at subsequent periods all low-value buyers offer this price.

Proposition 2 also yields conclusions concerning the order in which trading patterns arise. ( $\mathrm{P} 2.4 .2)$ establishes that if at date $t$ a positive proportion of sellers offer the low-value-buyer reservation price, then at every subsequent date all sellers offer this price (i.e., $z_{t}^{S L}>0$ implies $z_{t+1}^{S L}=1$ ). Hence the S, S-PS, and PS trading patterns (when they arise) precede the PS-P and $\mathrm{P}$ trading patterns. Then $(\mathrm{P} 2.3 .2)$ implies that the $\mathrm{S}$ trading pattern precedes all the other trading patterns (because $z_{t}^{L S}>0$ implies $z_{t+1}^{L S}=1$ ), and that the S-PS mixed trading pattern precedes PS. (P2.4.2) implies that the PS-P mixed trading pattern precedes $\mathbf{P}$. Hence, trading patterns arise in the following order: S, S-PS, PS, PS-P, and P. Of course, an equilibrium may exhibit only some of these patterns. In particular, the mixed trading patterns may be skipped (although the subset of the parameter space where all market equilibria exhibit mixed trading patterns is not negligible).

\section{Market Composition}

The market composition and the trading patterns intertwine. In both the $\mathrm{S}$ and the PS trading patterns, as well as in mixed trading patterns S-PS and PS-P the proportion of high-value buyers in the market is falling: in $\mathrm{S}$, each period a fraction $\alpha$ of high-value buyers exits the market, while no low-value buyer exits; in PS a fraction $\alpha$ of high-value buyers and a fraction $\frac{\alpha}{2}$ of low-value buyers exit the market each period. In the trading patterns $\mathbf{P}$ the same fraction $\alpha$ of each type of buyer exits the market at each date, and hence the proportion of high-value buyers in the market remains constant. Thus, the proportion of high-value buyers in the market decreases (quickly in S, and more slowly in PS), but once $P$ is reached, it becomes stationary. Proposition 3 establishes that trading patterns and the dynamics of market composition are governed by the relation of the proportion of high-value buyers in the market to the critical threshold $\pi^{*}=\left(u^{L}-c\right) /\left(u^{H}-c\right)$

Proposition 3. Assume that $c<u^{L}$. Let $(p, r, \lambda)$ be a market equilibrium and let $\bar{t} \in\{0, \ldots, T\}$.

The critical threshold $\left(\pi^{*}\right)$ :

(P3.1.1) If $b_{\bar{t}}^{H}<\pi^{*}$, then $p_{\bar{t}_{j}}^{S_{j}}=r_{\bar{t}}^{L}$ for every $j \leqslant n^{S}$ and $b_{\bar{t}+1}^{H}=b_{\bar{t}}^{H}$.

(P3.1.2) If $b_{\bar{t}}^{H}=\pi^{*}$, then $p_{\bar{i}}^{L_{i}}=r_{\bar{t}}^{S}$ for every $i \leqslant n^{L}$, and either

(i) $b_{i+1}^{H}<b_{\bar{t}}^{H}$, or

(ii) $b_{\bar{t}+1}^{H}=b_{\bar{i}}^{H}$, and $p_{t}^{S_{j}}=r_{t}^{L}$ for every $j \leqslant n^{S}$ and $t \geqslant \bar{t}$.

(P3.1.3) If $b_{\bar{i}}^{H}>\pi^{*}$, then $p_{\bar{i}}^{S_{j}}=r_{\bar{i}}^{H}$ for every $j \leqslant n^{S}$, and $b_{\bar{t}+1}^{H}<b_{\bar{t}}^{H}$. 
The critical threshold is eventually reached:

(P3.2) There is $\bar{T}=\bar{T}\left(b_{0}^{H}, \alpha, \pi^{*}\right)$ such that if $T>\bar{T}$, then $b_{t}^{H} \leqslant \pi^{*}$ for $t \geqslant \bar{T}$.

Proposition 3 establishes that if $b_{t}^{H}<\pi^{*}$, then the trading pattern at $t$ and subsequent periods is $\mathrm{P}(\mathrm{P} 3.1 .1)$. If $b_{t}^{H}>\pi^{*}$, then $z_{t}^{S L}=0$, and therefore the trading pattern at $t$ (and prior to $t$ ) is either S, a S-PS, or PS (P3.1.3). When $b_{t}^{H}=\pi^{*}$ the trading pattern at $t$ is either PS, a PS-P, or $P$ but it is $\mathbf{P}$ at every subsequent period (P3.1.2). If the time horizon is sufficiently long, then the proportion of high-value buyers in the market is eventually less than or equal to $\pi^{*}(\mathrm{P} 3.2)$, and therefore by (P3.1.1) and (P3.1.2) the trading pattern is eventually $\mathbf{P}$ and remains $P$.

\section{Efficiency and Delay}

In the low-cost case the unique efficient sequence of trading patterns is $\mathrm{P}$ at every date; i.e., $z_{t}^{H S}=z_{t}^{S H}=z_{t}^{L S}=z_{t}^{S L}=1$ for all $t$. Hence, by (P3.1.1) the sequence of equilibrium trading patterns is efficient whenever $b_{0}^{H}<\pi^{*}$. If $b_{0}^{H}>\pi^{*}$, however, the trading pattern at date 0 , and possibly afterward, is either S or PS, and therefore the sequence of equilibrium trading patterns is inefficient. Furthermore, since the critical threshold $\pi^{*}$ does not depend on either the discount factor or the time horizon, if $b_{0}^{H}>\pi^{*}$ the sequence of equilibrium trading patterns is inefficient even as frictions vanish. (Proposition 4 below, however, establishes that the welfare loss due to inefficient trading approaches zero as frictions vanish.)

Figure 3 shows an equilibrium for a market that opens for 10 periods and whose parameter values are those specified. In this equilibrium all three pure trading patterns arise. The top graph in Fig. 3 shows transaction prices. The trading pattern is S for periods 0 to 2 . It is PS for periods 3 to 7. In period 8 and 9 the good goes on "sale" (as sellers switch from offering the high-value-buyer reservation price to the low-value-buyer reservation price) and the trading pattern is $P$. The bottom graph shows the evolution of the market composition and the ratio $\pi_{t}$.

The set of competitive prices for the market in Fig. 3 is the interval $[0.2,0.4]$. We focus on the competitive price of 0.3 , since in a market equilibrium all transactions are at this price as frictions vanish (see Proposition 4). Table II shows the distribution of the surplus in four different settings: (i) at the (static) competitive equilibrium when price is 0.3 ; (ii) under efficient trading when price is 0.3 ; (iii) in the market equilibrium displayed in Fig. 3; and (iv) in the market equilibrium when information is complete (we comment on this last example in Section 4). Interestingly, in the market equilibrium displayed in Fig. 3 sellers capture more than twice the surplus than they capture in the competitive equilibrium. The market equilibrium is not efficient, since low-value buyers do not trade when matched 


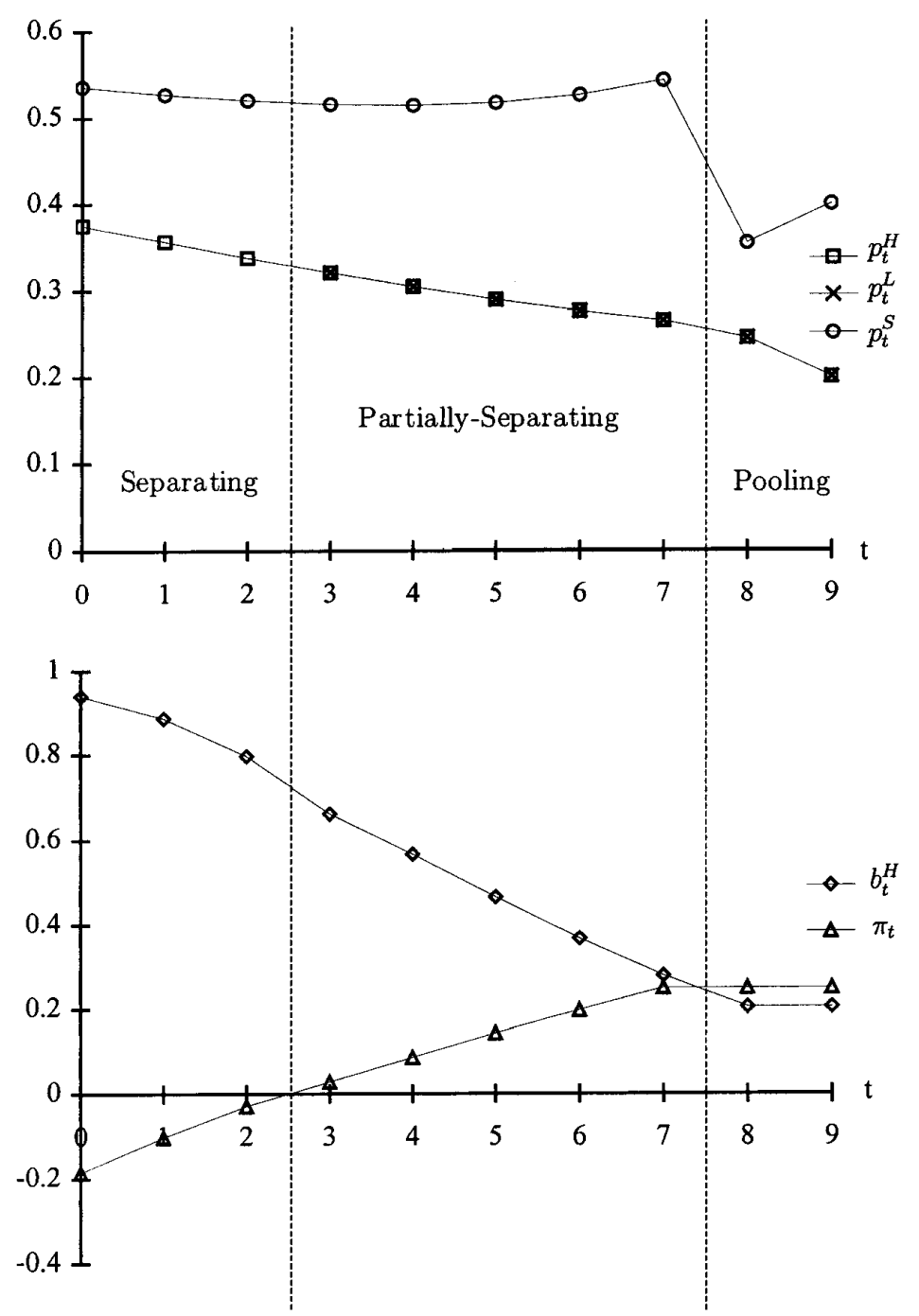

FIG. 3. Equilibrium in the low-cost case $\left(b_{0}^{H}=0.94, u^{H}=1, u^{L}=0.4, c=0.2, \delta=0.9, \alpha=0.5\right)$.

in periods 0 through 2 and trade only if they propose in periods 3 through 7 . The efficiency loss is small $(0.0042=0.6943-0.6901)$ since only $6 \%$ of the buyers are low-value at the market open. In this example, low-value buyers and sellers experience delay. Conditional on trading, a low-value buyer remains in the market an average of 6.18 periods in equilibrium, whereas he would remain 
TABLE II

The Division of Surplus

\begin{tabular}{lcccc}
\hline & \multicolumn{3}{c}{ Surplus } & \\
\cline { 2 - 4 } \multicolumn{1}{c}{ Trading } & High value & Low value & Seller & \multirow{2}{*}{ Total } \\
\hline Competitive & $0.6580(86 \%)$ & $0.0060(0.7 \%)$ & $0.1000(13 \%)$ & 0.7640 \\
Efficient & $0.5980(86 \%)$ & $0.0055(0.7 \%)$ & $0.0909(13 \%)$ & 0.6943 \\
Incomp. info. & $0.4740(69 \%)$ & $0.0033(0.4 \%)$ & $0.2128(30 \%)$ & 0.6901 \\
Comp. info. & $0.4353(63 \%)$ & $0.0018(0.2 \%)$ & $0.2526(36 \%)$ & 0.6897 \\
\hline
\end{tabular}

in the market an average of only 1.99 periods under efficient trading. The length of the delay experienced by sellers is small because high-value buyers are abundant $\left(b_{0}^{H}=0.96\right)$ and they trade with no delay.

\section{Market Equilibria as Friction Vanish}

Proposition 4 below establishes that as frictions vanish (i) transaction prices converge to the competitive equilibrium price $p^{*}=\left(u^{L}+c\right) / 2$, (ii) each trader receives his competitive equilibrium utility, and (iii) the surplus approaches the unconstrained surplus (equal to $b_{0}^{H}\left(u^{H}-c\right)+$ $\left(1-b_{0}^{H}\right)\left(u^{L}-c\right)$ in this case). For each $\delta \in(0,1)$ and $T$, denote by $r(\delta, T)$ the set of all sequences of equilibrium reservation prices, and by $V(\delta, T)$ the set of all sequences of equilibrium expected utilities. (Since a market equilibrium always exists, these sets are nonempty.) Also denote by $G(\delta, T)$ the set of all possible surpluses realized in equilibrium (i.e., the set of surpluses associated with the equilibrium trading patterns).

Proposition 4. Assume that $c<u^{L}$.

(P4.1) If $T<\infty$, then for every $\bar{t} \in\{0, \ldots, T\}$ :

(P4.1.1) $\lim _{\delta \rightarrow 1} \lim _{T \rightarrow \infty} r_{i}^{\tau}(\delta, T)=\lim _{T \rightarrow \infty} \lim _{\delta \rightarrow 1} r_{i}^{\tau}(\delta, T)=p^{*}$ for $\tau \in$ $\{H, L, S\}$.

(P4.1.2) $\lim _{\delta \rightarrow 1} \lim _{T \rightarrow \infty} V_{\bar{t}}^{\tau}(\delta, T)=\lim _{T \rightarrow \infty} \lim _{\delta \rightarrow 1} V_{\bar{t}}^{\tau}(\delta, T)=u^{\tau}-p^{*}$ for $\tau \in\{H, L\} ; \lim _{\delta \rightarrow 1} \lim _{T \rightarrow \infty} V_{\bar{i}}^{S}(\delta, T)=\lim _{T \rightarrow \infty} \lim _{\delta \rightarrow 1} V_{\bar{i}}^{S}(\delta, T)=$ $p^{*}-c$.

(P4.1.3) $\lim _{\delta \rightarrow 1} \lim _{T \rightarrow \infty} G(\delta, T)=\lim _{T \rightarrow \infty} \lim _{\delta \rightarrow 1} G(\delta, T)=b_{0}^{H}\left(u^{H}-c\right)$ $+b_{0}^{L}\left(u^{L}-c\right)$.

(P4.2) If $T=\infty$, then for every $\bar{t} \in\{0, \ldots, T\}$ :

(P4.2.1) $\lim _{\delta \rightarrow 1} r_{t}^{\tau}(\delta, T)=p^{*}$ for $\tau \in\{H, L, S\}$.

(P4.2.2) $\lim _{\delta \rightarrow 1} V_{\bar{t}}^{\tau}(\delta, T)=u^{\tau}-p^{*}$, for $\tau \in\{H, L\}$, and $\lim _{\delta \rightarrow 1} V_{\tilde{t}}^{S}(\delta, T)$ $=p^{*}-c$.

(P4.2.3) $\lim _{\delta \rightarrow 1} G(\delta, T)=b_{0}^{H}\left(u^{H}-c\right)+b_{0}^{L}\left(u^{L}-c\right)$. 
Although transaction prices converge to a competitive price, delay persists even as market frictions vanish and, in this sense, the market outcome is not competitive. Consider a market in which $b_{0}^{H}>\pi^{*}$ and let $(p, r, \lambda)$ be a market equilibrium. By Proposition 3 the trading pattern is either $\mathrm{S}$, S-PS, or PS at the market open. Define the sequence $\left\{\underline{b}_{t}^{H}\right\}$ as $\underline{b}_{0}^{H}=b_{0}^{H}$ and, for $t \geqslant 0$

$$
\underline{b}_{t+1}^{H}=\frac{(1-\alpha) \underline{b}_{t}^{H}}{(1-\alpha) \underline{b}_{t}^{H}+1-\underline{b}_{t}^{H}}
$$

The sequence $\left\{\underline{b}_{t}^{H}\right\}$ describes the evolution of the market composition as though the trading pattern is always $S$. Since the proportion of high-value buyers in the market falls more quickly in $\mathrm{S}$ than in the other trading patterns, then $b_{t}^{H} \geqslant \underline{b}_{t}^{H}$ for all $t \geqslant 0$. Therefore, if $\underline{t}$ is the smallest integer such that $\underline{b}_{t}^{H} \leqslant \pi^{*}$, then we have $b_{t}^{H}>\pi^{*}$ for $t<\underline{t}$; hence the trading pattern is either $\mathrm{S}$, an S-PS, or PS for periods 0 through $\underline{t}-1$. Thus, low-value buyers and sellers experience delay.

Since $\underline{t}$ is independent of the time horizon and the discount factor, delay persists even as frictions vanish. The surplus, however, approaches the unconstrained surplus, and therefore the welfare loss of inefficient trading approaches zero as friction vanish. This is a consequence of the fact that the length of delay is bounded (because the number of periods before the trading pattern is always pooling is bounded above, see (P3.2)), and therefore the cost of delay approaches zero as frictions vanish.

\section{MODELING ISSUES AND EXTENSIONS}

We analyze a market with one-sided incomplete information and equal measures of buyers and sellers. Our setup, however, can accommodate other markets. In this section we discussion some interesting extensions.

\subsection{Complete Information}

Under complete information (i.e., when sellers observe buyers' values) sellers may condition their price offers on the buyer's value. Our model can be readily modified to accommodate this possibility. It is easy to show that in the high-cost case the analog of Proposition 1 holds. In the low-cost case, the main difference is that the PS trading pattern never arises; lowvalue buyers and sellers trade whenever $r_{t}^{L}>r_{t}^{S}$, because sellers can discriminate between high- and low-value buyers. As the example in Fig. 4 shows, the $\mathrm{S}$, the $\mathrm{P}$, and the even mixed trading patterns (when $r_{t}^{L}=r_{t}^{S}$ ) may 


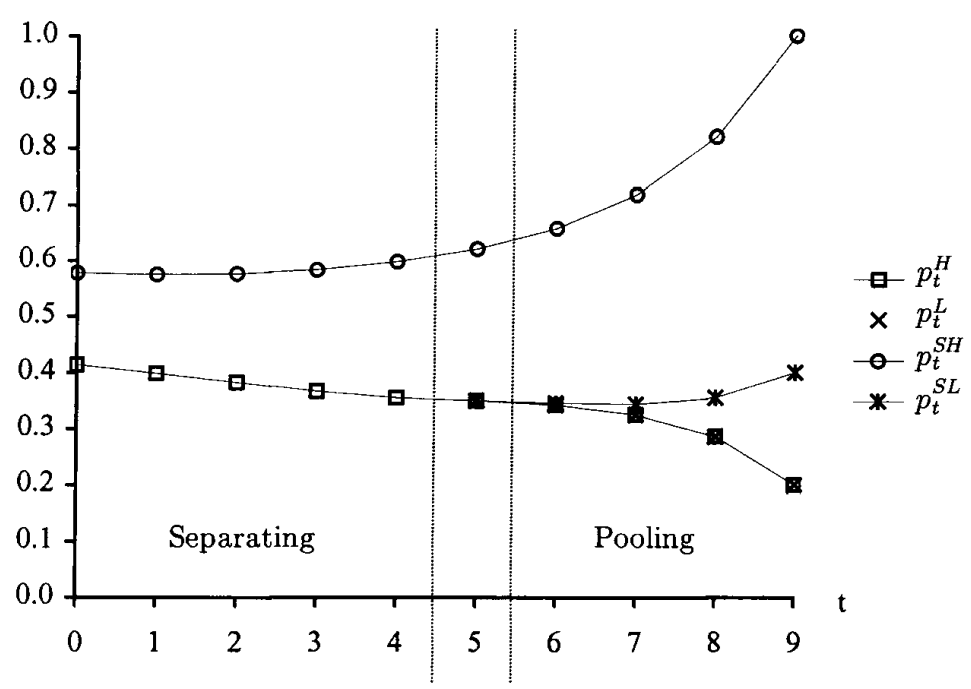

FIG. 4. Equilibrium in the low-cost case-complete information $\left(b_{0}^{H}=0.94, u^{H}=1\right.$, $\left.u^{L}=0.4, c=0.2, \delta=0.9, \alpha=0.5\right)$.

arise in equilibrium. In Fig. 4 seller price offers to high-value and low-value buyers are denoted by $p_{t}^{S L}$ and $p_{t}^{S H}$ respectively.

The parameter values for this market are the same as in Fig. 3, but information is now complete. The trading pattern is $S$ in periods $0-4$, it is mixed at period 5, and it is $P$ thereafter. High-value buyers are worse off when information is complete than when it is incomplete (see Table II) because they no longer trade at "sale" prices (i.e., at the low-value buyer reservation price). Sellers are better off because even in the pooling trading pattern they trade at the high-value buyer reservation price when proposing to high-value buyers, and because they trade with no delay with low-value buyers whenever $r_{t}^{L}>r_{t}^{S}$. Low-value buyers are worse off because, except in the last period, sellers have higher reservation prices. Perhaps surprisingly, the surplus realized in equilibrium is less when information is complete $(0.6897)$ than when it is incomplete (0.6901). It is easy to construct examples in which the opposite inequality holds. Hence, whereas heterogeneity is clearly a source of inefficiency, the presence of asymmetric information may enhance efficiency.

\subsection{Unequal Measures}

Our assumption that buyers and sellers are present in the market in equal measures does not seem to be a significant restriction as unequal measures can be equalized by introducing a positive measure of a nontrading 
(a)

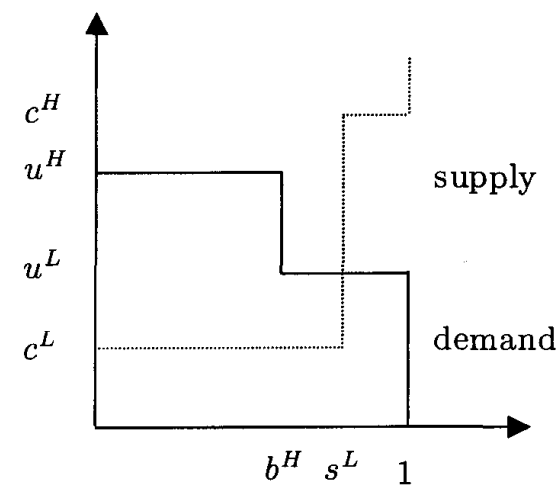

(b)

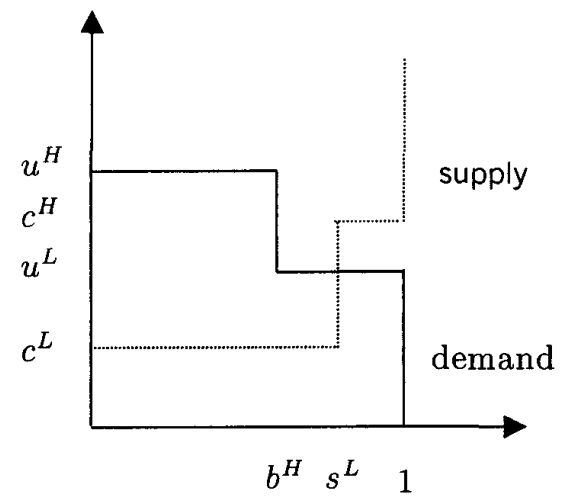

FIG. 5. (a) Two-sided uncertainty $\left(c^{H}>u^{H}\right)$. (b) Two-sided uncertainty $\left(c^{H}<u^{H}\right)$.

type. For example, the low-cost case in which there is a measure 1 of buyers and a measure $s^{L}$ of sellers, with $s^{L}<1$, can be modelled by introducing a measure $1-s^{L}$ of sellers, each with unit cost $c^{H}$, where $c^{H}$ is greater than $u^{H}$, so that supply and demand are as given in Fig. 5a. By the same reasoning, our model of the high-cost case can be interpreted as a model of the situation where (high-value) buyers are present in the market in smaller measure than sellers (and low-value buyers are a nontrading type introduced to equalize measures). Introducing new types, however, renders the analysis more cumbersome when it makes the information uncertainty "two-sided."

\subsection{Two-Sided Incomplete Information}

When uncertainty is two-sided as in Figs. 5a and 5b, the evolution of the market composition at each date is described by the proportion of 
high-value buyers $\left(b_{t}^{H}\right)$ and low-cost sellers $\left(s_{t}^{L}\right)$, which are determined endogenously by the trading patterns. In addition, the number of feasible (pure) trading patterns increases significantly (a trading pattern now is represented by an 8-dimensional vector).

The example in Fig. 6 suggests the features of market equilibrium when uncertainty is two-sided. In the example, supply and demand are qualitatively the same as in Fig. 5b. For simplicity, the example is developed for the case $\alpha=1$ and $\delta=1$, so that the only friction present is the finite horizon. We denote by $p_{t}^{H B}\left(r_{t}^{H B}\right)$ the price offer (reservation price) of high-value buyers at time $t$. Price offers and reservation prices for low-value buyers (LB), high-cost sellers (HS) and low-cost sellers (LS) are denoted analogously.

The graph in Fig. 6 shows transaction prices. The table below the graph gives $b_{t}^{H}, s_{t}^{L}, \pi_{t}^{b}$ and $\pi_{t}^{s}$ for periods 0 through 9 , where $\pi_{t}^{b}=$ $\left(r_{t}^{L B}-r_{t}^{L S}\right) /\left(r_{t}^{H B}-r_{t}^{L S}\right)$ and $\pi_{t}^{s}=\left(r_{t}^{H B}-r_{t}^{H S}\right) /\left(r_{t}^{H B}-r_{t}^{L S}\right)$. The vertical lines in Fig. 6 separate distinct trading patterns. Equilibrium price offers depend upon the relation of $b_{t}^{H}$ to $\pi_{t}^{b}, s_{t}^{L}$ to $\pi_{t}^{s}$, and the sign of $\pi_{t}^{s}$ and $\pi_{t}^{b}$. For example, whether a high-value buyer offers the high or low-cost seller reservation price depends on the relation of $s_{t}^{L}$ to $\pi_{t}^{s}$. Since $r_{t}^{H S}>r_{t}^{L S}$, a highvalue buyer obtains $r_{t}^{H B}-r_{t}^{H S}$ if he offers $r_{t}^{H S}$ (this offer is accepted by both types of sellers); he obtains the expected gain of $s_{t}^{L}\left(r_{t}^{H B}-r_{t}^{L S}\right)$ if he offers $r_{t}^{L S}$ (this offer is accepted only by low-cost sellers). Hence high-value buyers offer $r_{t}^{L S}$ if $s_{t}^{L}>\pi_{t}^{s}$ and offer $r_{t}^{H S}$ if $s_{t}^{L}<\pi_{t}^{s}$. In the example, high-value buyers offer $r_{t}^{L S}$ in periods 0 to 4 and offer $r_{t}^{H S}$ in periods 5 to 9 . Whether low-value buyers offer $r_{t}^{L S}$ or make a smaller (rejected) price offer depends on the sign of $\pi_{t}^{b}$. If $\pi_{t}^{b}>0$ then $r_{t}^{L B}>r_{t}^{L S}$, and low-value buyers offer $r_{t}^{L S}$; otherwise they make price offers which are rejected. In the example, lowvalue buyers make price offers which are rejected in periods 0 to 2 , but offer the low-cost seller reservation price thereafter. Similarly, low-cost sellers offer $r_{t}^{H B}$ if $b_{t}^{H}>\pi_{t}^{b}$, and $r_{t}^{L B}$ if $b_{t}^{H}<\pi_{t}^{b}$; high-cost sellers offer $r_{t}^{H B}$ if $\pi_{t}^{s}>0$, and make rejected offers otherwise.

Qualitatively, the trading patterns of this example are similar to the lowcost case: high-value buyers and low-cost sellers always trade; in periods 0 to 2 there is no other trade; from period 3 on, low-value buyers trade when they propose to low-cost sellers; from period 4 on, high-cost sellers trade when they propose to high-value buyers; from periods 5 to 9 all matches (except those between low-value buyers and high-cost sellers) end with trade. In equilibrium the average price received by sellers is 0.4164 , which is close to 0.4 , the competitive price. Note that in period 4 low-cost sellers trade with both types of buyers, and hence in later periods only high-cost sellers remain (i.e., $s_{t}^{L}=0$ ). In period 5 high-value buyers trade with both types of sellers, and hence in later periods only low-value buyers remain. Thus, after period 5 there is no trade, since only low-value buyers and high-cost sellers remain in the market. 


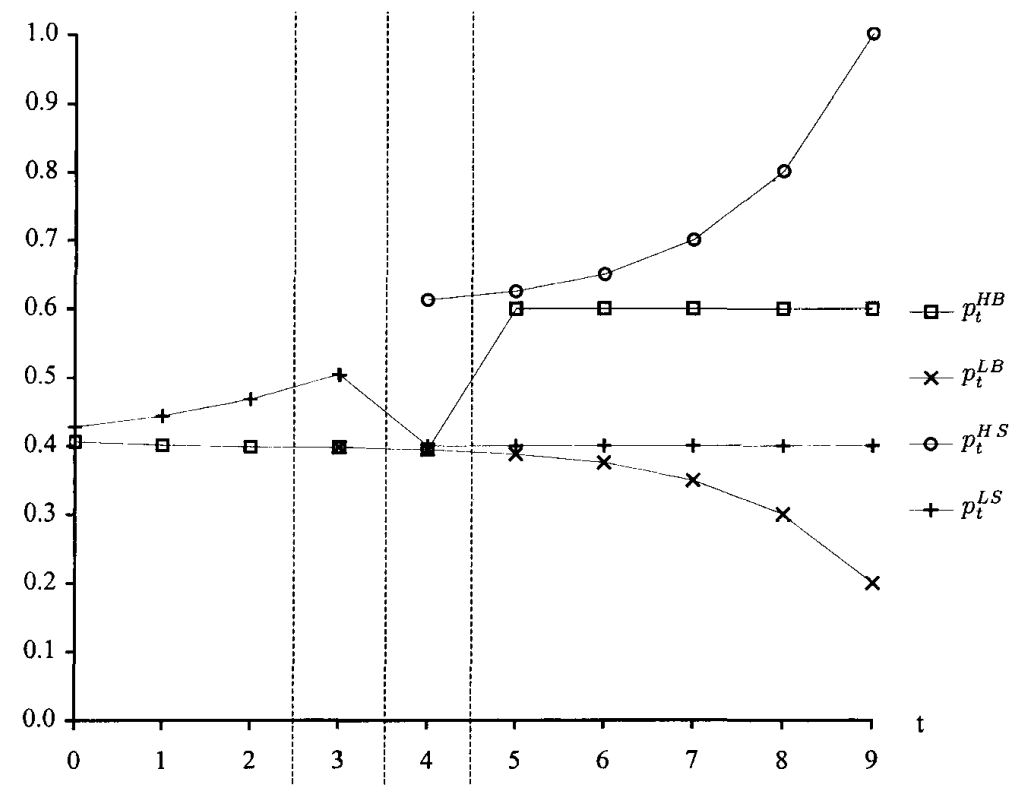

$\begin{array}{lllllllllll}b_{t}^{H} & .940 & .293 & .074 & .024 & .012 & .006 & .000 & .000 & .000 & .000 \\ s_{t}^{L} & .980 & .746 & .691 & .675 & .503 & .000 & .000 & .000 & .000 & .000 \\ & & & & & & & & & \\ \pi_{t}^{b} & -.369 & -.068 & -.005 & .013 & .026 & .053 & .091 & .143 & .200 & .250 \\ \pi_{t}^{s}-7.887 & -3.630 & -1.896 & -.897 & .057 & .105 & .182 & .286 & .400 & .500\end{array}$

FIG. 6. Equilibrium with two-sided uncertainty $\left(b_{0}^{H}=0.94, s_{0}^{L}=0.98, u^{H}=1, u^{L}=0.4\right.$, $\left.c^{H}=0.6, c^{L}=0.2, \delta=\alpha=1\right)$.

As this example illustrates, our model is easily modified to accommodate the two-sided incomplete information case. However, the introduction of new endogenous variables and the enlargement of the set of possible trading patterns makes the study of this case considerable more complex. An analysis of the trading patterns, dynamics, efficiency, and the asymptotic properties of market equilibria for this case is an important topic for future research.

\section{APPENDIX: PROOFS OF PROPOSITIONS 1 TO 4}

We begin stating a number of lemmas - see Moreno and Wooders [5] for proofs. Throughout assume that $(p, r, \lambda)$ is a market equilibrium. 
Lemmata. $\forall t \in\{0, \ldots, T\}$ :

1. $\forall \tau \in\{H, L, S\}, \forall k, k^{\prime} \in\left\{1, \ldots, n^{\tau}\right\}$ :

(L1.1) $r_{t}^{\tau_{k}}=r_{t}^{\tau_{\alpha^{\prime}}}$;

(L1.2) $\quad R_{t}^{\tau_{k}}=R_{t}^{\tau_{t^{\prime}}}$;

(L1.3) $\quad P_{t}^{\tau_{k}}=P_{t}^{\tau_{t}^{\prime}}$;

(L1.4) $\quad V_{t}^{\tau_{k}}=V_{t}^{\tau_{k^{\prime}}}$.

2. $\forall \tau \in\{H, L\}, \forall i \leqslant n^{\tau}$.

(L2.1) $p_{t}^{\tau_{i} \leqslant r_{t}^{S}}$;

(L2.2) $r_{t}^{\tau}>r_{t}^{S} \Rightarrow p_{t}^{\tau_{i}}=r_{t}^{S}$;

(L2.3) $r_{t}^{\tau}<r_{t}^{S} \Rightarrow p_{t}^{\tau_{i}}<r_{t}^{S}$.

3. If $r_{t}^{H}>\max \left\{r_{t}^{L}, r_{t}^{S}\right\}$ then $\forall j \leqslant n^{S}$ :

(L3.1) $p_{t}^{S_{j}} \in\left\{r_{t}^{L}, r_{t}^{H}\right\}$;

(L3.2) $b_{t}^{H}<\pi_{t} \Rightarrow p_{t}^{S_{j}}=r_{t}^{L}$;

(L3.3) $b_{t}^{H}=\pi_{t} \Rightarrow P_{t}^{S}\left(r_{t}^{H}\right)=P_{t}^{S}\left(r_{t}^{L}\right)$;

(L3.4) $b_{t}^{H}>\pi_{t} \Rightarrow p_{t}^{S_{j}}=r_{t}^{H}$.

4. (L4.1) $r_{t}^{H}>r_{t}^{L}$;

(L4.2) $V_{t}^{H}-V_{t}^{L} \leqslant \alpha\left(u^{H}-u^{L}\right) \frac{1-\delta^{T-t+1}(1-\alpha)^{T-t+1}}{1-\delta(1-\alpha)}$.

5. (L5.1) $r_{t}^{H}>r_{t}^{S}$;

(L5.2) $\quad V_{t}^{H}+V_{t}^{S} \leqslant \alpha\left(u^{H}-c\right) \frac{1-\delta^{T-t+1}(1-\alpha)^{T-t+1}}{1-\delta(1-\alpha)}$

(L5.3) $b_{t}^{H} V_{t}^{H}+b_{t}^{L} V_{t}^{L}+V_{t}^{S} \leqslant \alpha\left(b_{t}^{H} u^{H}+b_{t}^{L} u^{L}-c\right) \frac{1-\delta^{T-t+1}(1-\alpha)^{T-t+1}}{1-\delta(1-\alpha)}$.

6. (L6.1) $p_{t}^{H_{i}}=r_{t}^{S}, \forall i \leqslant n^{H}$;

(L6.2) $z_{t}^{H_{i}}=1, \forall_{i} \leqslant n^{H}$;

(L6.3) $P_{t}^{H}=u^{H}-r_{t}^{s}$;

(L6.4) $R_{t}^{L}=u^{L}-r_{t}^{L}$;

(L6.5) $R_{t}^{S}=r_{t}^{S}-c$.

7. The sequence $\left\{b_{t}^{H}\right\}_{t=0}^{T+1}$ is decreasing.

For 8 to 12 assume that $c<u^{L}$.

8. (L8.1) $p_{t}^{S_{j}}=r_{t}^{L} \forall j \leqslant n^{S}$ and $\pi_{t}=\pi^{*} \Rightarrow \pi_{t-1}=\pi^{*}$;

(L8.2) $p_{t}^{S_{j}}=r_{t}^{L} \forall j \leqslant n^{S}$ and $\pi_{t}<\pi^{*} \Rightarrow \pi_{t-1}<\pi^{*}$. 
9. (L9.1) $b_{t}^{H} \geqslant \pi^{*} \geqslant \pi_{t} \Rightarrow \pi^{*} \geqslant \pi_{t-1}$;

(L9.2) $b_{t}^{H}>\pi^{*} \geqslant \pi_{t} \Rightarrow \pi^{*}>\pi_{t-1}$;

(L9.3) $b_{t}^{H} \geqslant \pi^{*} \geqslant \pi_{t}$ and $p_{t}^{S_{j}}=r_{t}^{H}$ for some $j \leqslant n^{S} \Rightarrow \pi^{*}>\pi_{t-1}$.

10. Assume that there is $\hat{T} \leqslant T$ such that $\pi^{*}=\pi_{t}$ for $t \in\{\hat{T}, \ldots, T\}$ :

(L10.1) $b_{t}^{H}<\pi^{*} \Rightarrow \pi^{*}=\pi_{s}$ for $s \geqslant t$

(L10.2) $b_{t}^{H}=\pi^{*} \Rightarrow$ either $b_{t+1}^{H}<\pi^{*}$ or $b_{s}^{H}=\pi^{*}$ for $s \geqslant t$;

(L10.3) $b_{t}^{H}>\pi^{*} \Rightarrow \pi^{*} \geqslant \pi_{s}$ for $s \leqslant t$.

11. Assume $T<\infty$. There is $\varepsilon(\alpha, T)>0$ such that if $\delta \in[1-\varepsilon(\alpha, T), 1]$, then $r_{t}^{L}-r_{t}^{S} \leqslant 0 \Rightarrow r_{t-1}^{L}-r_{t-1}^{S}<0$.

12. Assume $T=\infty$. There is $\widetilde{T}<\infty$ such that for $t \geqslant \tilde{T}: z_{t}^{H S}=z_{t}^{S H}=z_{t}^{L S}=$ $z_{t}^{S L}=1$ (i.e., the trading pattern is pooling), $r_{t}^{\tau}=r^{\tau} \forall \tau \in\{H, L, S\}$, and $\pi_{t}=\pi^{*}$.

We are now ready to prove Propositions 1 to 4 .

Proof of Proposition 1. (P1.1.1) holds by (L1.1), and (P1.1.2) follows from (L5.1) and the fact that $r_{t}^{S} \geqslant c>u^{L} \geqslant r_{t}^{L}$ for $t \in\{0, \ldots, T\}$. Also (P1.2) is implied by (P1.1.2), (L2.2), (L2.3), and (L3.4). In order to prove (P1.3), note that (P1.2) implies $I\left(p_{t}^{L_{i}}, r_{t}^{S}\right)=I\left(r_{t}^{L}, p_{t}^{S_{j}}\right)=0$ for $i \leqslant n^{L}, j \leqslant n^{S}$ and $t \in\{0, \ldots, T\}$; therefore $z_{t}^{L_{i}}=0$ for $i \leqslant n^{L}$ and $t \in\{0, \ldots, T\}$. Also since $z_{t}^{H_{i}}=1$ for $i \leqslant n^{H}$ and $t \in\{0, \ldots, T\}$ by (L6.2), we have

$$
\begin{aligned}
b_{t}^{H} & =\frac{b_{t-1}^{H}\left(1-\alpha z_{t-1}^{H}\right)}{b_{t-1}^{\bar{H}}\left(1-\alpha z_{t-1}^{H}\right)+b_{t-1}^{L}\left(1-\alpha z_{t-1}^{L}\right)} \\
& =\frac{(1-\alpha) b_{t-1}^{H}}{(1-\alpha) b_{t-1}^{H}+1-b_{t-1}^{H}}<b_{t-1}^{H} .
\end{aligned}
$$

As for (P1.4), the surplus realized in equilibrium can be readily calculated from (S.1)-(S.2). Note that (P1.2) implies that the equilibrium trading patterns, $\bar{z}$, satisfy $\bar{z}_{t}^{H S}=\bar{z}_{t}^{S H}=\bar{z}_{t}^{H}=1$ and $\bar{z}_{t}^{L S}=\bar{z}_{t}^{S L}=\bar{z}_{t}^{L}=0$. Hence (S.2) yields $g^{L}(\delta, T, \bar{z})=0$, and

$$
\begin{aligned}
g^{H}(\delta, T, \bar{z}) & =\alpha+\delta(1-\alpha) \alpha+\cdots+\delta^{T}(1-\alpha)^{T} \alpha \\
& =\frac{\alpha\left(1-\delta^{T+1}(1-\alpha)^{T+1}\right)}{1-\delta(1-\alpha)} .
\end{aligned}
$$


Substituting in (S.1) yields

$$
G(\delta, T)=G(\delta, T, \bar{z})=b_{0}^{H}\left(u^{H}-c\right) \frac{\alpha\left(1-\delta^{T+1}(1-\alpha)^{T+1}\right)}{1-\delta(1-\alpha)},
$$

which establishes (P1.4). (P1.5.2) and (P1.6.2) follow immediately.

We prove (P1.5.1). All transactions are at either the high-value-buyer or the seller reservation price. These prices are determined, for $t<T$, by the system of difference equations

$$
\left[\begin{array}{c}
r_{t}^{H} \\
r_{t}^{S}
\end{array}\right]=(1-\delta)\left[\begin{array}{c}
u^{H} \\
c
\end{array}\right]+\delta\left[\begin{array}{cc}
1-\frac{\alpha}{2} & \frac{\alpha}{2} \\
\frac{\alpha}{2} b_{t+1}^{H} & 1-\frac{\alpha}{2} b_{t+1}^{H}
\end{array}\right]\left[\begin{array}{c}
r_{t+1}^{H} \\
r_{t+1}^{S}
\end{array}\right]
$$

Thus, since $1-\frac{\alpha}{2}\left(1+b_{k}^{H}\right) \leqslant 1-\frac{\alpha}{2}$ for each $k$, we have

$$
\begin{aligned}
r_{t}^{H}-r_{t}^{S} & =(1-\delta)\left(u^{H}-c\right)+\left[1-\frac{\alpha}{2}\left(1-b_{t+1}^{H}\right)\right]\left(r_{t+1}^{H}-r_{t+1}^{S}\right) \\
& \leqslant\left(u^{H}-c\right)\left[(1-\delta) \frac{1-\delta^{T-t}\left(1-\frac{\alpha}{2}\right)^{T-t}}{1-\delta\left(1-\frac{\alpha}{2}\right)}+\delta^{T-t}\left(1-\frac{\alpha}{2}\right)^{T-t}\right] .
\end{aligned}
$$

Also from above we have for each $t$

$$
b_{t}^{H}=\frac{(1-\alpha) b_{t-1}^{H}}{(1-\alpha) b_{t-1}^{H}+1-b_{t-1}^{H}}=\frac{(1-\alpha)^{t} b_{0}^{H}}{1-\left[1-(1-\alpha)^{t}\right] b_{0}^{H}}
$$
Since $\sum_{k=0}^{T-t} \delta^{k} \leqslant \frac{1}{1-\delta}, \frac{1}{1-\left[1-(1-\alpha)^{k}\right] b_{0}^{H}}<\frac{1}{1-b_{0}^{H}}$, and $(1-\alpha)^{k}<\left(1-\frac{\alpha}{2}\right)^{k}$ for
$k>0$,

$$
\begin{aligned}
r_{t}^{S} & =(1-\delta) c+\delta\left[r_{t+1}^{S}+\frac{\alpha}{2} b_{t+1}^{H}\left(r_{t+1}^{H}-r_{t+1}^{S}\right)\right] \\
& =c+\frac{\alpha}{2} \sum_{k=t+1}^{T} \delta^{k-t} b_{k}^{H}\left(r_{k}^{H}-r_{k}^{S}\right) \\
& <c+\frac{\alpha}{2}\left(u^{H}-c\right) \frac{b_{0}^{H}}{\left(1-b_{0}^{H}\right)\left[1-\delta\left(1-\frac{\alpha}{2}\right)\right]} \eta_{t}(\delta, T),
\end{aligned}
$$


where

$$
\eta_{t}(\delta, T)=\sum_{k=t+1}^{T} \delta^{k-t}\left(1-\frac{\alpha}{2}\right)^{k}\left[1-\delta+\frac{\alpha}{2} \delta^{T-k+1}\left(1-\frac{\alpha}{2}\right)^{T-k}\right]
$$

If $T<\infty$, then

$$
\begin{aligned}
\eta_{t}(\delta, T)= & (1-\delta) \delta\left(1-\frac{\alpha}{2}\right)^{t+1} \frac{1-\delta^{T-t}\left(1-\frac{\alpha}{2}\right)^{T-t}}{1-\delta\left(1-\frac{\alpha}{2}\right)} \\
& +\frac{\alpha}{2}\left(1-\frac{\alpha}{2}\right)^{T}(T-t) \delta^{T-t+1}
\end{aligned}
$$

and since $\lim _{T \rightarrow \infty}(T-t)\left(1-\frac{\alpha}{2}\right)^{T}=0$, we have

$$
\lim _{T \rightarrow \infty} \lim _{\delta \rightarrow 1} \eta_{t}(\delta, T)=\lim _{\delta \rightarrow 1} \lim _{T \rightarrow \infty} \eta_{t}(\delta, T)=0
$$

If $T=\infty$, then

$$
\lim _{\delta \rightarrow 1} \eta_{t}(\delta, T)=\lim _{\delta \rightarrow 1}(1-\delta) \delta\left(1-\frac{\alpha}{2}\right)^{t+1} \frac{1}{1-\delta\left(1-\frac{\alpha}{2}\right)}=0
$$

Since $r_{t}^{S} \geqslant c$, if $T<\infty$ we have

$$
\lim _{T \rightarrow \infty} \lim _{\delta \rightarrow 1} r_{t}^{S}=\lim _{\delta \rightarrow 1} \lim _{T \rightarrow \infty} r_{t}^{S}=c
$$

and if $T=\infty$ we have $\lim _{\delta \rightarrow 1} r_{t}^{S}=c$. Also, the above inequality and (P1.1.2) imply

$$
r_{t}^{S}<r_{t}^{H} \leqslant\left(u^{H}-c\right)\left[(1-\delta) \frac{1-\delta^{T-t}\left(1-\frac{\alpha}{2}\right)^{T-t}}{1-\delta\left(1-\frac{\alpha}{2}\right)}+\delta^{T-t}\left(1-\frac{\alpha}{2}\right)^{T-t}\right]+r_{t}^{S}
$$


Hence if $T<\infty$ we have

$$
\lim _{T \rightarrow \infty} \lim _{\delta \rightarrow 1} r_{t}^{H}=\lim _{\delta \rightarrow 1} \lim _{T \rightarrow \infty} r_{t}^{H}=c ;
$$

and if $T=\infty$ we have $\lim _{\delta \rightarrow 1} r_{t}^{H}=c$. Therefore, (P1.5.1) and (P1.6.1) hold.

Proof of Proposition 2. (P2.1.1) is (L1.1); (P2.1.2) is implied by (L4.1) and (L5.1); (P2.2) is (L6.1); (P2.3.1) is contained in (L2.1); (P2.4.1) is implied by (P2.1.2) and (L3.1).

We prove (P2.3.2). Assume that $T<\infty$. As $V_{T+1}^{H}=V_{T+1}^{L}=V_{T+1}^{S}=0$, by Lemma 11 there is $\varepsilon(\alpha, T)>0$ such that if $\delta>1-\varepsilon(\alpha, T)$, then $r_{t}^{L} \leqslant r_{t}^{S}$ implies $r_{t-1}^{L}<r_{t-1}^{S}$. Suppose that $\delta \in[1-\varepsilon(\alpha, T), 1]$. If $p_{\bar{t}}^{L_{i}}<r_{\bar{t}}^{S}$ for some $i \leqslant n^{L}$, then (L2.2) implies $r_{\tilde{t}}^{L} \leqslant r_{t}^{S}$; hence $r_{\bar{t}-1}^{L}<r_{\tilde{t}-1}^{S}$, and by induction $r_{t}^{L}<r_{t}^{S}$ for $t<\bar{t}$; therefore (L2.3) implies $p_{t}^{L_{i}}<r_{t}^{S}$ for every $t<\bar{t}$ and $i \leqslant n^{L}$. This establishes (i) of (P2.3.2). Now assume that $p_{i_{i} i^{\prime}}^{L^{\prime}}=r_{i}^{S}$ for some $i^{\prime} \leqslant n^{L}$, and suppose by way of contradiction that $p_{\hat{t}^{\prime}}^{L{ }^{\prime}} \neq r_{\hat{t}}^{S}$ for some $i^{\prime \prime} \leqslant n^{L}$ and $\hat{t}>\bar{t}$. Then $p_{\hat{t}}^{L_{i}{ }^{\prime \prime}}<r_{\hat{t}}^{S}$ by (L2.1). The previous argument implies $p_{t}^{L_{i}}<r_{t}^{S}$ for every $t<\hat{t}$ and $i \leqslant n^{L}$; in particular, $p_{t_{i}}^{L^{\prime}}<r_{\bar{t}}^{S}$, which is a contradiction.

We now prove $(\mathbf{P} 2.4 .2)$. If $T<\infty$, since $V_{T+1}^{H}=V_{T+1}^{L}=V_{T+1}^{S}=0$, we have $r_{T}^{H}=u^{H}, r_{T}^{L}=u^{L}$, and $r_{T}^{S}=c$; therefore $\pi_{T}=\pi^{*}$, and hence Lemma 10 applies. Assume that $p_{\bar{t}}^{S_{j}}=r_{\bar{t}}^{L}$ for some $j \leqslant n^{S}$. Then we have $b_{\bar{t}}^{H} \leqslant \pi^{*}$, for if $b_{\bar{t}}^{H}>\pi^{*}$ then $b_{\bar{t}}^{H}>\pi_{\bar{t}}$ by (L10.3), and therefore we would have $p_{\bar{t}^{j}}^{S_{i}}=r_{\bar{t}}^{H}$ for each $j \leqslant n^{S}$ by (L3.4) and (P2.1.2), which is a contradiction. Suppose that $b_{\bar{t}}^{H}<\pi^{*}$; then $\pi_{t}=\pi^{*}$ for each $t \geqslant \bar{t}$ by (L10.1), and therefore Lemma 7 implies $b_{t}^{H}<\pi_{t}$ for each $t \geqslant \bar{t}$. Thus $p_{t}^{S_{j}}=r_{t}^{L}$ for every $t \geqslant \bar{t}$ and $j \leqslant n^{S}$ by (L3.2). Suppose that $b_{\bar{t}}^{H}=\pi^{*}$; then either $b_{\bar{t}+1}^{H}<\pi^{*}$ or $b_{t}^{H}=\pi^{*}$ for $t \geqslant \bar{t}$ by (L10.2). If $b_{\bar{i}+1}^{H}<\pi^{*}$, then (L10.1) again implies $b_{t}^{H}<\pi_{t}$ for each $t \geqslant \bar{t}+1$ and therefore $p_{t}^{S_{j}}=r_{t}^{L}$ for every $t \geqslant \bar{t}+1$ and $j \leqslant n^{S}$, by (L3.2). If $b_{t}^{H}=\pi^{*}$ for $t \geqslant \bar{t}$, since high-value buyers always trade when they are matched (by P2.2, $\mathbf{P} 2.1$.2, and P2.4.1), then low-value buyers must also trade when matched; hence $p_{t}^{S_{j}}=r_{t}^{L}$ for every $t \geqslant \bar{t}+1$ and $j \leqslant n^{S}$. If $T=\infty$, then there is $\hat{T}$ such that $\pi_{\hat{T}}=\pi^{*}$ by (L12.4), and therefore the same arguments apply.

We establish (P2.4.3). Suppose by way of contradiction that $p_{\bar{t}^{\prime}}^{S^{\prime}}=r_{\bar{t}}^{H}$ for some $j^{\prime} \leqslant n^{S}$, and $p_{\hat{t}^{\prime}}^{S^{n}}=r_{\hat{i}}^{L}$ for some $j^{\prime \prime} \leqslant n^{S}$ and $\hat{t}<\bar{t}$. Then (P2.4.2) implies $p_{t}^{S_{j}}=r_{t}^{L}$ for every $t>\hat{t}$ and $j \leqslant n^{S}$. In particular, $p_{\hat{t}^{\prime}}^{S^{\prime}}=r_{\hat{t}}^{L}$, which is a contradiction.

Finally, we prove (P2.5). If $p_{\tilde{t}}^{S_{j}}=r_{\tilde{t}}^{L}$ for some $j \leqslant n^{S}$ then (L3.4) implies $b_{\bar{t}}^{H} \leqslant \pi_{i}$, and since $b_{\bar{t}}^{H}>0$ and $r_{i}^{H}-r_{i}^{S}>0$, by (L5.1), we have $r_{\bar{t}}^{L}-r_{i}^{S}>0$. Hence $p_{\bar{t}}^{L_{i}}=r_{\bar{t}}^{S}$ for every $i \leqslant n^{L}$ by (L2.2).

Proof of Proposition 3. If $T<\infty$, since $V_{T+1}^{H}=V_{T+1}^{L}=V_{T+1}^{S}=0$, we have $\pi_{T}=\pi^{*}$. If $T=\infty$, then by Lemma 12 there is $\hat{T}$ such that $\pi_{t}=\pi^{*}$ for $t \geqslant \hat{T}$. Hence the assumptions of Lemma 10 hold. 
We prove (P3.1.1). Assume $b_{\bar{t}}^{H}<\pi^{*}$; then $b_{\bar{t}}^{H}<\pi_{\bar{t}}$ by (L10.1), and therefore $p_{\frac{S_{j}}{S^{\prime}}}=r_{\bar{t}}^{L}$ for $j \leqslant n^{S}$ by (L3.2); hence $p_{i}^{L_{i}}=r_{\bar{t}}^{S}$ by (P2.5) for every $i \leqslant n^{L}$. Thus $I\left(p_{\bar{t}}^{L_{i}}, r_{i}^{S}\right)=I\left(r_{i}^{L}, p_{\tilde{t}_{j}}^{S_{j}}\right)=1$, and therefore

$$
z_{\bar{t}}^{L_{i}}=\frac{1}{2} \sum_{j=1}^{n^{S}} \lambda \lambda_{\bar{t}^{j}} I\left(p_{\bar{t}^{i}}^{L_{i}}, r_{\bar{t}}^{S}\right)+\frac{1}{2} \sum_{j=1}^{n^{S}} \lambda S_{\bar{t}}^{j} I\left(r_{\bar{t}}^{L}, p_{\bar{t}}^{S_{j}}\right)=1 .
$$

Since $z_{\bar{i}}^{H_{i}}=1$ for each $i \leqslant n^{H}$ by (L6.2), we have

$$
b_{i+1}^{H}=\frac{(1-\alpha) b_{i}^{H}}{(1-\alpha) b_{\bar{t}}^{H}+(1-\alpha) b_{\bar{t}}^{L}}=b_{\bar{t}}^{H} .
$$

We establish (P3.1.2). Assume $b_{\bar{t}}^{H}=\pi^{*}$. Then by (L10.2) either $b_{\bar{t}+1}^{H}<\pi^{*}$ or $b_{\bar{i}}^{H}=\cdots=b_{T}^{H}=\pi^{*}$. If $b_{\bar{i}+1}^{H}<\pi^{*}=b_{\bar{t}}^{H}$, then $b_{\bar{t}+1}^{H}<\pi_{\bar{t}+1}$ (by (L10.1)), and $p_{i^{j}+1}^{S_{1}}=r_{\tilde{i}+1}^{L}$ for every $j \leqslant n^{S}$ by (L3.2). Hence $\pi_{i}=\pi^{*}>0$ by (L8.1), and therefore $r_{\bar{t}}^{L}>r_{\bar{t}}^{S}$ (because $r_{\bar{t}}^{H}>r_{\bar{t}}^{S}$ by (P2.1.2)), and (L2.1) implies $p_{\bar{t}}^{L_{i}}=r_{\bar{t}}^{S}$ for every $i \leqslant n^{L}$. If $b_{\bar{t}}^{H}=\cdots=b_{T}^{H}=\pi^{*}$, since $z_{t}^{H}=1$, then $z_{t}^{L_{i}}=1$ for $i \leqslant n^{L}$ and $t \geqslant \bar{t}$; hence for $t \geqslant \bar{t}$ we have $p_{t}^{L_{i}}=r_{t}^{S}$ for $i \leqslant n^{L}$, and $p_{t}^{S_{j}}=r_{t}^{L}$ for $j \leqslant n^{S}$.

We show that (P3.1.3) holds. Suppose that $b_{\bar{i}}^{H}>\pi^{*}$; then $b_{\bar{i}}^{H}>\pi_{\bar{i}}$ by Lemma (L10.3), and therefore $p_{\bar{t}^{j}}=r_{\bar{t}}^{H}$ for each $j \leqslant n^{S}$ by (L3.4). Since $r_{\bar{i}}^{H}>r_{\bar{i}}^{L}$ by $(L 4.1)$, then $I\left(r_{\bar{t}}^{L}, p_{\bar{t}}^{S}\right)=0$ for each $j \leqslant n^{S}$, and therefore for each $i \leqslant n^{L}$ we have

$$
z_{\bar{t}}^{L_{i}}=\frac{1}{2} \sum_{j=1}^{n^{S}} \lambda_{\bar{t}}^{S_{j}} I\left(p_{\bar{t}}^{L}, r_{\bar{t}}^{S_{j}}\right) \leqslant \frac{1}{2}
$$

Thus, since $z_{\bar{t}}^{H}=1$ by (L6.2), we get

$$
b_{\bar{t}+1}^{H}=\frac{(1-\alpha) b_{\bar{t}}^{H}}{(1-\alpha) b_{\bar{t}}^{H}+b_{\bar{t}}^{L}\left(1-\alpha z_{\bar{t}}^{L}\right)} \leqslant \frac{(1-\alpha) b_{\bar{i}}^{H}}{(1-\alpha) b_{\bar{t}}^{H}+\left(1-\frac{\alpha}{2}\right)\left(1-b_{\bar{t}}^{H}\right)}<b_{\bar{i}}^{H} .
$$

Finally, we establish (P3.2). Since $\left\{b_{t}^{H}\right\}$ is a decreasing sequence by Lemma 7, it suffices to show that there is $\bar{T}$ such that $b_{\bar{T}}^{H} \leqslant \pi^{*}$. If $b_{0}^{H} \leqslant \pi^{*}$, take $\bar{T}=0$. Assume that $b_{0}^{H}>\pi^{*}$; w show that there is $\bar{T}=\bar{T}\left(b_{0}^{H}, \alpha, \pi^{*}\right)$ such that $b_{T}^{H}<\pi^{*}$. Define the sequence $\left\{\underline{b}_{t}\right\}$ by $\underline{b}_{0}=b_{0}^{H}$, and for $t>0$

$$
\begin{aligned}
\underline{b}_{t+1} & =\frac{(1-\alpha) \underline{b}_{t}}{(1-\alpha) \underline{b}_{t}+\left(1-\frac{\alpha}{2}\right)\left(1-\underline{b}_{t}\right)} \\
& =\frac{(1-\alpha)^{t+1} b_{0}^{H}}{(1-\alpha)^{t+1} b_{0}^{H}+\left(1-\frac{\alpha}{2}\right)^{t+1}\left(1-b_{0}^{H}\right)}
\end{aligned}
$$


We show that $b_{t}^{H}>\pi^{*}$ implies $b_{t+1}^{H} \leqslant \underline{b}_{t+1}$. Assume $b_{t}^{H}>\pi^{*}$; we show by induction that $b_{k}^{H} \leqslant \underline{b}_{k}$ for $k \leqslant t+1$. By construction $b_{0}^{H} \leqslant \underline{b}_{0}$. Assume that $b_{k}^{H} \leqslant \underline{b}_{k}$ for $k \leqslant t$; we show that $b_{k+1}^{H} \leqslant \underline{b}_{k+1}$. Since $\left\{b_{t}^{H}\right\}$ is nonincreasing by Lemma 7 and $k \leqslant t$, then $b_{k}^{H} \geqslant b_{t}^{H}>\pi^{*}$. Therefore (P3.1.3) implies $p_{k}^{S_{j}}=r_{k}^{H}$, and hence $I\left(r_{k}^{L}, p_{k}^{S_{j}}\right)=0$, for every $j \leqslant n^{S}$. Therefore $z_{k}^{L_{i} \leqslant \frac{1}{2} \text { for }}$ every $i \leqslant n^{L}$. Since $z_{k}^{H}=1$, we have

$$
\begin{aligned}
b_{k+1}^{H} & =\frac{(1-\alpha) b_{k}^{H}}{(1-\alpha) b_{k}^{H}+b_{k}^{L}\left(1-\alpha z_{k}^{L}\right)} \\
& \leqslant \frac{(1-\alpha) \underline{b}_{k}}{(1-\alpha) \underline{b}_{k}+\left(1-\frac{\alpha}{2}\right)\left(1-\underline{b}_{k}\right)}=\underline{b}_{k+1} .
\end{aligned}
$$

Let $\bar{T}=\bar{T}\left(b_{0}^{H}, \alpha, \pi^{*}\right)$ be the first integer such that $\underline{b}_{\bar{T}}<\pi^{*}$. (Such an integer exists since $\left\{\underline{b}_{t}\right\}$ converges to zero.) We show that $b_{\bar{T}}^{H} \leqslant \pi^{*}$. Suppose $b_{\bar{T}}^{H}>$ $\pi^{*}$; then $b_{\bar{T}-1}^{H}>\pi^{*}$ by Lemma 7 , and hence $b_{\bar{T}}^{\underline{H}} \leqslant \underline{b}_{\bar{T}}<\pi^{*}$, which is a contradiction. Hence (P3.2) holds.

Proof of Proposition 4. For each $\delta \in(0,1)$ and $T$, let $r \in r(\delta, T)$ be a sequence of equilibrium reservation prices and let $V$ be the corresponding sequence of expected utilities. By (P3.2) there is $\bar{T}=\bar{T}\left(b_{0}^{H}, \alpha, \pi^{*}\right)$ such that if $T>\bar{T}$, then $b_{t}^{H} \leqslant \pi^{*}$ for $t \geqslant \bar{T}$. Assume that $T>\bar{T}$. Thus, (P3.1.1) and (P3.1.2) imply $p_{t}^{S_{j}}=r_{t}^{L}<r_{t}^{H}$ for every $j \leqslant n^{S}$ and $t \geqslant \bar{T}+1$, and therefore $p_{t}^{L_{i}}=r_{t}^{S}$ for every $i \leqslant n^{L}$ and $t \geqslant \bar{T}+1$ by $(\mathrm{P} 2.5)$. Also $p_{t}^{H_{i}}=r_{t}^{S}$ for every $i \leqslant n^{H}$ and $t$ by (P2.2). Hence for $t \geqslant \bar{T}+1$ the trading pattern is pooling (i.e., $z_{t}^{L}=1$ for $t \geqslant \bar{T}+1$ ), and therefore traders' reservation prices for $t \geqslant \bar{T}+1$ are given by the system of difference equations

$$
\left[\begin{array}{c}
V_{t}^{H} \\
V_{t}^{L} \\
V_{t}^{S}
\end{array}\right]=\frac{\alpha}{2}\left[\begin{array}{c}
2 u^{H}-u^{L}-c \\
u^{L}-c \\
u^{L}-c
\end{array}\right]+\delta\left[\begin{array}{ccc}
1-\alpha & \frac{\alpha}{2} & -\frac{\alpha}{2} \\
0 & 1-\frac{\alpha}{2} & -\frac{\alpha}{2} \\
0 & -\frac{\alpha}{2} & 1-\frac{\alpha}{2}
\end{array}\right]\left[\begin{array}{c}
V_{t+1}^{H} \\
V_{t+1}^{L} \\
V_{t+1}^{S}
\end{array}\right]
$$

The solution of this system, whether $T$ is finite, and therefore $V_{T+1}^{H}=$ $V_{T+1}^{L}=V_{T+1}^{S}=0$, or $T$ is infinite, is

$$
V_{t}^{\tau}=\frac{\alpha}{2}\left(u^{L}-c\right) \frac{1-[\delta(1-\alpha)]^{T-t+1}}{1-\delta(1-\alpha)},
$$


for $\tau \in\{L, S\}$, and

$$
V_{t}^{H}=\frac{\alpha}{2}\left(2 u^{H}-u^{L}-c\right) \frac{1-[\delta(1-\alpha)]^{T-t+1}}{1-\delta(1-\alpha)} .
$$

Hence if $T<\infty$, for $t \geqslant \bar{T}+1$ we have

$$
\begin{aligned}
\lim _{\delta \rightarrow 1} \lim _{T \rightarrow \infty} V_{t}^{S} & =\lim _{T \rightarrow \infty} \lim _{\delta \rightarrow 1} V_{t}^{S}=\frac{u^{L}-c}{2} \\
& =\lim _{\delta \rightarrow 1} \lim _{T \rightarrow \infty} V_{t}^{L}=\lim _{T \rightarrow \infty} \lim _{\delta \rightarrow 1} V_{t}^{L},
\end{aligned}
$$

and

$$
\lim _{\delta \rightarrow 1} \lim _{T \rightarrow \infty} V_{t}^{H}=\lim _{T \rightarrow \infty} \lim _{\delta \rightarrow 1} V_{t}^{H}=u^{H}-\frac{u^{L}+c}{2} .
$$

If $T=\infty$, for $t \geqslant \bar{T}+1$ we have

$$
\lim _{\delta \rightarrow 1} V_{t}^{S}=\frac{u^{L}-c}{2}=\lim _{\delta \rightarrow 1} V_{t}^{L},
$$

and

$$
\lim _{\delta \rightarrow 1} V_{t}^{H}=u^{H}-\frac{u^{L}+c}{2} .
$$

Now let $t<\bar{T}+1$. A trader of type $\tau$ who is in the market at date $t$ obtains an expected utility of $V_{t}^{\tau}$ by following his equilibrium strategy; thus the expected utility to a trader who remains in the market at $t$ must satisfy $V_{t}^{\tau} \geqslant \delta^{\bar{T}+1-t} V_{\bar{T}+1}^{\tau}$, for otherwise he benefits from a deviation where he makes unacceptable offers and rejects any offers until date $\bar{T}+1$, following his equilibrium strategy thereafter. Also $V_{t}^{H}+V_{t}^{S} \leqslant u^{H}-c$ by (L5.2). Thus

$$
\delta^{\bar{T}+1-t} V_{T+1}^{S} \leqslant V_{t}^{S} \leqslant u^{H}-c-V_{t}^{H} \leqslant u^{H}-c-\delta^{\bar{T}+1-t} V_{T+1}^{H} .
$$

Also

$$
\delta^{\bar{T}+1-t} V_{\bar{T}+1}^{H} \leqslant V_{t}^{H} \leqslant u^{H}-c-V_{t}^{S} \leqslant u^{H}-c-\delta^{\bar{T}+1-t} V_{T+1}^{S} ;
$$

Therefore if $T<\infty$ we have

$$
\lim _{\delta \rightarrow 1} \lim _{T \rightarrow \infty} V_{t}^{S}=\lim _{T \rightarrow \infty} \lim _{\delta \rightarrow 1} V_{t}^{S}=\frac{u^{L}-c}{2},
$$


and

$$
\lim _{\delta \rightarrow 1} \lim _{T \rightarrow \infty} V_{t}^{H}=\lim _{T \rightarrow \infty} \lim _{\delta \rightarrow 1} V_{t}^{H}=u^{H}-\frac{u^{L}+c}{2} .
$$

Similarly, if $T=\infty$ we have

$$
\lim _{\delta \rightarrow 1} V_{t}^{S}=\frac{u^{L}-c}{2}
$$

and

$$
\lim _{\delta \rightarrow 1} V_{t}^{H}=u^{H}-\frac{u^{L}+c}{2}
$$

For low-value buyers, we can write the expected utility at time $t$ of a low-value buyer who follows the $i$ th low-value buyer strategy as

$$
\begin{aligned}
V_{t}^{L}= & \frac{\alpha}{2}\left[\left(u^{L}-p_{t}^{L_{i}}\right) I\left(p_{t}^{L_{i}}, r_{t}^{S}\right)+\delta V_{t+1}^{L}\left(1-I\left(p_{t}^{L_{i}}, r_{t}^{S}\right)\right)\right] \\
& +\frac{\alpha}{2} \sum_{j=1}^{n^{S}} \lambda_{t}^{S_{j}}\left[\left(u^{L}-p_{t}^{S_{j}}\right) I\left(r_{t}^{L}, p_{t}^{S_{j}}\right)+\delta V_{t+1}^{L}\left(1-I\left(r_{t}^{L}, p_{t}^{S_{j}}\right)\right)\right] \\
& +(1-\alpha) \delta V_{t+1}^{L} .
\end{aligned}
$$

Further, (P2.4.1) and (E.1) imply $\left(u^{L}-p_{t}^{S_{j}}\right) I\left(r_{t}^{L}, p_{t}^{S_{j}}\right)+\delta V_{t+1}^{L}(1-$ $\left.I\left(r_{t}^{L}, p_{t}^{S_{j}}\right)\right)=\delta V_{t+1}^{L}$; and therefore

$$
\begin{aligned}
V_{t}^{L}= & \frac{\alpha}{2}\left[\left(u^{L}-r_{t}^{L}+r_{t}^{L}-p_{t}^{L_{i}}\right) I\left(p_{t}^{L_{i}}, r_{t}^{S}\right)+\delta V_{t+1}^{L}\left(1-I\left(p_{t}^{L_{i}}, r_{t}^{S}\right)\right)\right] \\
& +\left(1-\frac{\alpha}{2}\right) \delta V_{t+1}^{L} \\
= & \frac{\alpha}{2}\left(r_{t}^{L}-p_{t}^{L_{i}}\right) I\left(p_{t}^{L_{i}}, r_{t}^{S}\right)+\delta V_{t+1}^{L}
\end{aligned}
$$

Thus, by (L2.1) - (L2.3) and (E.1) this can be rewritten as

$$
\begin{aligned}
V_{t}^{L} & =\frac{\alpha}{2} \max \left\{r_{t}^{L}-r_{t}^{S}, 0\right\}+\delta V_{t+1}^{L} \\
& =\frac{\alpha}{2} \max \left\{u^{L}-c-\delta\left(V_{t+1}^{L}+V_{t+1}^{S}\right), 0\right\}+\delta V_{t+1}^{L}
\end{aligned}
$$


Assume $T<\infty$. If

$$
\lim _{\delta \rightarrow 1} \lim _{T \rightarrow \infty} V_{t+1}^{L}=\lim _{T \rightarrow \infty} \lim _{\delta \rightarrow 1} V_{t+1}^{L}=\frac{u^{L}-c}{2}
$$

then

$$
\begin{aligned}
\lim _{\delta \rightarrow 1} \lim _{T \rightarrow \infty} V_{t}^{L}= & \frac{\alpha}{2} \max \left\{u^{L}-c-\lim _{\delta \rightarrow 1} \lim _{T \rightarrow \infty} \delta V_{t+1}^{L}-\lim _{\delta \rightarrow 1} \lim _{T \rightarrow \infty} \delta V_{t+1}^{S}, 0\right\} \\
& +\lim _{\delta \rightarrow 1} \lim _{T \rightarrow \infty} \delta V_{t+1}^{L} \\
= & \frac{u^{L}-c}{2}
\end{aligned}
$$

and

$$
\begin{aligned}
\lim _{T \rightarrow \infty} \lim _{\delta \rightarrow 1} V_{t}^{L}= & \frac{\alpha}{2} \max \left\{u^{L}-c-\lim _{T \rightarrow \infty} \lim _{\delta \rightarrow 1} \delta V_{t+1}^{L}-\lim _{T \rightarrow \infty} \lim _{\delta \rightarrow 1} \delta V_{t+1}^{S}, 0\right\} \\
& +\lim _{T \rightarrow \infty} \lim _{\delta \rightarrow 1} \delta V_{t+1}^{L} \\
= & \frac{u^{L}-c}{2} .
\end{aligned}
$$

Since

$$
\begin{aligned}
\lim _{\delta \rightarrow 1} \lim _{T \rightarrow \infty} V_{\bar{T}+1}^{L} & =\lim _{\delta \rightarrow 1} \lim _{T \rightarrow \infty} \frac{\alpha}{2}\left(u^{L}-c\right) \frac{1-[\delta(1-\alpha)]^{T-\bar{T}}}{1-\delta(1-\alpha)} \\
& =\frac{u^{L}-c}{2}=\lim _{T \rightarrow \infty} \lim _{\delta \rightarrow 1} V_{\bar{T}+1}^{L}
\end{aligned}
$$

induction yields

$$
\lim _{\delta \rightarrow 1} \lim _{T \rightarrow \infty} V_{t}^{L}=\lim _{T \rightarrow \infty} \lim _{\delta \rightarrow 1} V_{t}^{L}=\frac{u^{L}-c}{2}
$$

for $t<\bar{T}+1$. An analogous argument shows that if $T=\infty$, then

$$
\lim _{\delta \rightarrow 1} V_{t}^{L}=\frac{u^{L}-c}{2}
$$

for $t<\bar{T}+1$. Hence (P4.1.2) and (P4.2.2) hold. 
Now we prove (P4.1.1) and (P4.2.1). By (E.1) we have $r_{t}^{S}=c+\delta V_{t+1}^{S}$ and $r_{t}^{\tau}=u^{t}-\delta V_{t+1}^{\tau}$ for $\tau \in\{H, L\}$. Therefore if $T<\infty$ the above limits imply

$$
\lim _{\delta \rightarrow 1} \lim _{T \rightarrow \infty} r_{t}^{\tau}=\lim _{T \rightarrow \infty} \lim _{\delta \rightarrow 1} r_{t}^{\tau}=\frac{u^{L}+c}{2}
$$

for $\tau \in\{H, L, S\}$ and $t \in\{0, \ldots, T\}$. If $T=\infty$, then

$$
\lim _{\delta \rightarrow 1} r_{t}^{\tau}=\frac{u^{L}+c}{2}
$$

for $\tau \in\{H, L, S\}$, and $t \in\{0, \ldots, T\}$.

Finally, given a sequence of equilibrium trading patterns, $z$, the surplus realized can be calculated from Eqs. (S.1) and (S.2) in Section 2. Since $z_{t}^{H}=1$ by (L6.2), we have

$$
g^{H}(\delta, T, z)=\frac{\alpha\left(1-\delta^{T+1}(1-\alpha)^{T+1}\right)}{1-\delta(1-\alpha)} .
$$

Also for $t>\bar{T}=\bar{T}\left(b_{0}^{H}, \alpha, \pi^{*}\right)$ the trading pattern is pooling, i.e., $z_{t}^{L}=1$, and hence for $T>\bar{T}$ we have

$$
\begin{aligned}
g^{L}(\delta, T, z)= & \alpha z_{0}^{L}+\delta\left(1-\alpha z_{0}^{L}\right) \alpha z_{1}^{L}+\cdots \\
& +\delta^{\bar{T}}\left(1-\alpha z_{0}^{L}\right)\left(1-\alpha z_{1}^{L}\right) \cdots\left(1-\alpha z_{\bar{T}-1}^{L}\right) \alpha z_{\bar{T}}^{L} \\
& +\delta^{\bar{T}+1}\left(1-\alpha z_{0}^{L}\right)\left(1-\alpha z_{1}^{L}\right) \cdots \\
& \times\left(1-\alpha z_{\bar{T}}^{L}\right) \frac{\alpha\left(1-\delta^{T-\bar{T}}(1-\alpha)^{T-\bar{T}}\right)}{1-\delta(1-\alpha)}
\end{aligned}
$$

Hence if $T<\infty$ we have

$$
\lim _{\delta \rightarrow 1} \lim _{T \rightarrow \infty} g^{\tau}(\delta, T, z)=\lim _{T \rightarrow \infty} \lim _{\delta \rightarrow 1} g^{\tau}(\delta, T, z)=1,
$$

for $\tau \in\{H, L\}$, and therefore

$$
\lim _{\delta \rightarrow 1} \lim _{T \rightarrow \infty} G(\delta, T, z)=\lim _{T \rightarrow \infty} \lim _{\delta \rightarrow 1} G(\delta, T, z)=b_{0}^{H}\left(u^{H}-c\right)+b_{0}^{L}\left(u^{L}-c\right) .
$$

If $T=\infty$, then

$$
\lim _{\delta \rightarrow 1} g^{L}(\delta, T, z)=\lim _{\delta \rightarrow 1} g^{H}(\delta, T, z)=1
$$


and therefore

$\lim _{\delta \rightarrow 1} G(\delta, T, z)=b_{0}^{H}\left(u^{H}-c\right)+b_{0}^{L}\left(u^{L}-c\right)$.

Therefore (P4.1.3) and (P4.2.3) hold.

\section{REFERENCES}

1. K. Binmore and M. Herrero, Matching and bargaining in dynamic markets, Rev. Econ. Stud. 55 (1988), 17-31.

2. D. Gale, Limit theorems for markets with sequential bargaining, J. Econ. Theory 43 (1987), 20-54.

3. O. Hart and J. Tirole, Contract renegotiation and coasian dynamics, Rev. Econ. Stud. 55 (1988), 509-540.

4. M. Jackson and T. Palfrey, Efficiency and voluntary implementation in markets with repeated pairwise bargaining, Econometrica 66 (1999), 1353-1388.

5. D. Moreno and J. Wooders, Prices, delay, and the dynamics of trade, Working Paper 99-32, Universidad Carlos III de Madrid, 1999.

6. M. Osborne and A. Rubinstein, "Bargaining and Markets," Academic Press, New York, 1990.

7. M. Peters, Ex ante price offers in a matching game: Non steady states, Econometrica $\mathbf{5 9}$ (1991), 1425-1454.

8. A. Rubinstein and A. Wolinsky, Equilibrium in a market with sequential bargaining, Econometrica 53 (1985), 1133-1150.

9. L. Samuelson, Disagreement in markets with matching and bargaining, Rev. Econ. Stud. 59 (1992), 177-186.

10. M. Sattinger, Search and the efficient assignment of workers to jobs, Int. Econ. Rev. 36 (1995), 283-302.

11. R. Serrano and $O$. Yosha, Welfare analysis of a market with pairwise meetings and asymmetric information, Econ. Theory 8 (1996), 167-175.

12. R. Serrano and $O$. Yosha, Decentralized information and the walrasian outcome: $A$ pairwise meetings market with private values, Mimeo, 1996

13. H. Varian, A model of sales, Amer. Econ. Rev. 70 (1980), 651-659.

14. A. Wolinsky, Information revelation in a market with pairwise meetings, Econometrica 58 (1990), 1-23. 